\title{
Effects of IL-7 on memory CD8+ $T$ cell homeostasis are influenced by the timing of therapy in mice
}

\author{
Som G. Nanjappa, ${ }^{1}$ Jane H. Walent, ${ }^{1}$ Michel Morre, ${ }^{2}$ and M. Suresh ${ }^{1}$ \\ ${ }^{1}$ Department of Pathobiological Sciences, University of Wisconsin-Madison, Madison, Wisconsin, USA. ${ }^{2}$ Cytheris Inc., Issy-Les-Moulineaux, France.
}

\begin{abstract}
IL-7 is integral to the generation and maintenance of $\mathrm{CD8}^{+} \mathrm{T}$ cell memory, and insufficient IL-7 is believed to limit survival and the persistence of memory $\mathrm{CD8}^{+} \mathrm{T}$ cells. Here, we show that during the mouse $\mathrm{T}$ cell response to lymphocytic choriomeningitis virus, IL-7 enhanced the number of memory CD8 ${ }^{+} \mathrm{T}$ cells when its administration was restricted to the contraction phase of the response. Likewise, IL-7 administration during the contraction phase of the mouse $T$ cell response to vaccinia virus or a DNA vaccine potentiated antigen-specific $\mathrm{CD8}^{+}$memory $\mathrm{T}$ cell proliferation and function. Qualitatively, CD8 ${ }^{+} \mathrm{T}$ cells from IL-7-treated mice exhibited superior recall responses and improved viral control. IL-7 treatment during the memory phase stimulated a marked increase in the number of memory $\mathrm{CD8}^{+} \mathrm{T}$ cells, but the effects were transient. IL-7 therapy during contraction of the secondary $\mathrm{CD8}^{+} \mathrm{T}$ cell response also expanded the pool of memory $\mathrm{CD8}^{+} \mathrm{T}$ cells. Collectively, our studies show differential effects of IL-7 on memory $\mathrm{CD8}^{+} \mathrm{T}$ cell homeostasis and underscore the importance of the timing of IL-7 therapy to effectively improve $\mathrm{CD8}^{+} \mathrm{T}$ cell memory and protective immunity. These findings may have implications in the clinical use of IL-7 as an immunotherapeutic agent to bolster vaccineinduced $\mathrm{CD}^{+} \mathrm{T}$ cell memory.
\end{abstract}

\section{Introduction}

It is well recognized that CD8 $\mathrm{T}$ cells play an important role in defense against several viral, intracellular bacterial, and protozoan infections. Therefore, for vaccines to be effective against these agents, they need to elicit potent CD8 T cell memory (1). The development of protective immunity depends on the elicitation of a threshold number of functional memory CD8 T cells (2), and the development of effective vaccines to protect against chronic infections (e.g., HIV, hepatitis B virus, and hepatitis C virus) has been a formidable challenge to immunologists. Hence, there is an increased sense of urgency to develop rational immunotherapies to boost vaccine-induced CD8 T cell memory. In recent years, there has been a surge in our understanding of the molecular and cellular basis of CD8 T cell memory. Cytokines that use receptors of the common cytokine receptor $\gamma$-chain family - IL-2, IL-7, and IL-15 in particular - regulate T cell responses and play a crucial role in the generation and/or maintenance of CD8 T cell memory (3). Of these cytokines, IL-7 has emerged as a "master regulator" of peripheral $\mathrm{T}$ cell homeostasis (4). Apart from its role in thymic T cell development, IL-7 is essential for survival of naive $\mathrm{T}$ cells in the periphery. Following antigenic stimulation, the primary expansion of CD8 T cells might not require IL-7, but the subsequent survival and differentiation into memory $T$ cells is dependent on IL-7R signaling (5-7). Elegant studies have recently shown that IL-7R ${ }^{+v e}$, but not IL-7R-ve, effector CD8 T cells preferentially give rise to long-lived memory cells $(8,9)$. Furthermore, IL-7 promotes the proliferative renewal and long-term maintenance of memory CD8 T cells (10).

Nonstandard abbreviations used: Arm, Armstrong 53b; CDK, cyclin-dependent kinase; GP, glycoprotein; LCMV, lymphocytic choriomeningitis virus; NP, nucleoprotein; $\mathrm{PCMV}$, plasmid containing the $\mathrm{CMV}$ promoter; $\mathrm{VV}$, vaccinia virus.

Conflict of interest: The authors have declared that no conflict of interest exists. Citation for this article: J. Clin. Invest. 118:1027-1039 (2008). doi:10.1172/JCI32020.
Because IL-7 is integral to the survival of naive and memory $\mathrm{T}$ cells, it is not surprising that several human clinical trials are in progress to evaluate the therapeutic benefits of IL-7 administration in chronic viral infections, cancer, and lymphopenia (11-14). Mice and primates tolerate IL-7 therapy with no detectable side effects, and IL-7 treatment in animal models of cancer and chronic viral infection has provided encouraging results (15-17). IL-7 treatment of primates infected with SIV has been reported to induce a transient increase in the number of naive and memory phenotype $T$ cells without affecting viral titers $(15,16)$. In other studies, IL-7 treatment of $\mathrm{T}$ cell-depleted mice improved the development of immune competence, and administration of IL-7 to mice all through the expansion and contraction improved protective immunity against tumors (17). Multiple stromal tissues present in the thymus, bone marrow, and other lymphoid organs produce low level of IL-7 constitutively, and overexpression of IL-7 has been shown to increase the pool of memory CD8 T cells, which suggests that the restricted availability of IL-7 might limit the survival and persistence of memory CD8 T cells (18). Despite the promise that IL-7 could be used to enhance immunity, the optimal treatment regimen has not been determined yet, nor have the factors that regulate the consequences of IL-7 administration been extensively examined. A thorough understanding of the factors that influence the effects of IL-7 therapy is essential before clinical use of IL-7 becomes a reality. Here, we show that the effectiveness of IL-7 therapy in enhancing CD8 T cell memory depends on the phase of the CD8 T cell response. Treatment of mice with IL-7 during the contraction phase and not expansion phase enhanced the magnitude of CD8 T cell memory following an acute lymphocytic choriomeningitis virus (LCMV) infection. Likewise, IL-7 treatment during the contraction phase of the CD8 $\mathrm{T}$ cell response to vaccinia virus $(\mathrm{VV})$ or DNA vaccine induced a substantive increase in the number of memory CD8 T cells. Moreover, in mice immunized with a DNA vaccine, IL-7 treatment augmented recall responses of CD8 $\mathrm{T}$ cells and improved viral control following challenge with a 

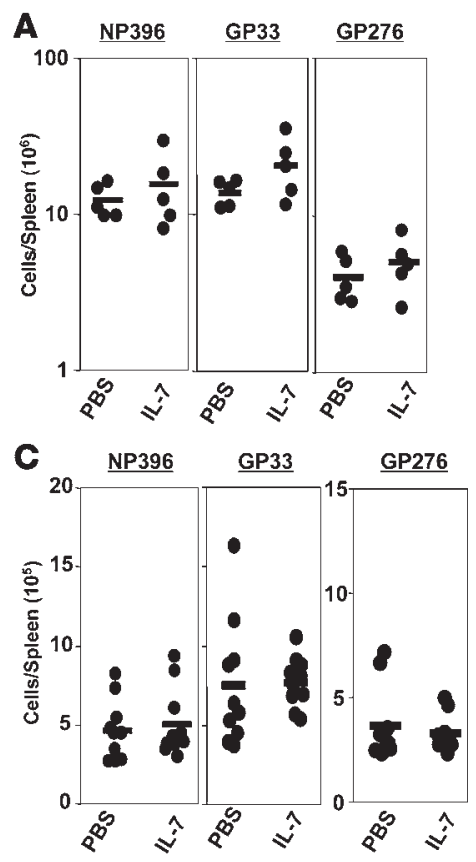

B
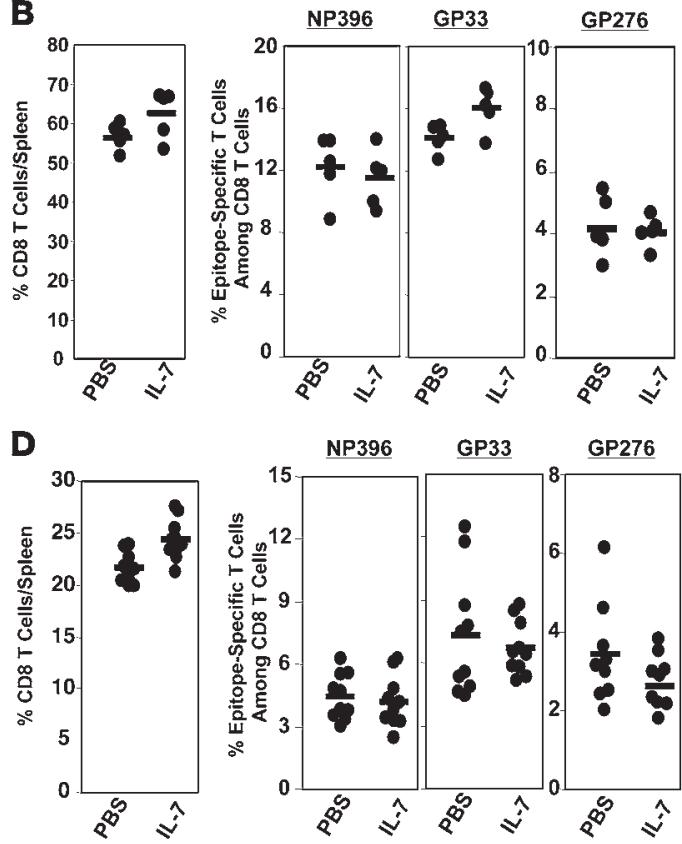

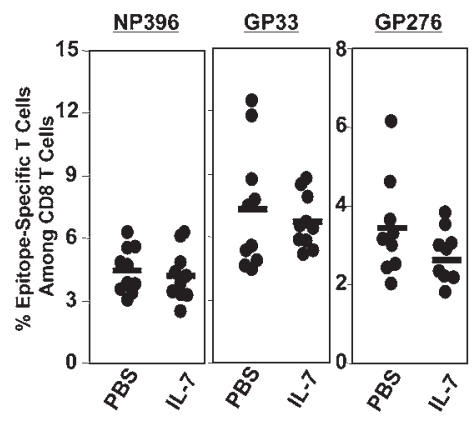

Figure 1

IL-7 treatment during the expansion phase of the CD8 $\mathrm{T}$ cell response. Cohorts of LCMV-infected C57BL/6 mice were treated daily with IL-7 or vehicle control PBS between days 1 and 7 after infection. On days 8 (A and $\mathbf{B})$ and 41 (C and $\mathbf{D})$ after infection, CD8 T cells in the spleen specific to the indicated LCMV epitopes were quantitated by intracellular staining for IFN- $\gamma$. Each symbol represents data from the spleens of individual mice. virulent strain of LCMV. Treatment of mice with IL-7 during the memory phase markedly increased the number of memory CD8 T cells, at least in the short term. Additionally, IL-7 administration during the contraction phase of a secondary CD8 $\mathrm{T}$ cell response led to significant improvement in the size of CD8 T cell memory. Collectively, our studies show differential effects of IL-7 on CD8 T cell memory, which depend on the timing of treatment in relation to the phase of the CD8 T cell response. These findings have clear implications in the rational use of IL-7 as an immunotherapeutic agent to bolster vaccine-induced CD8 T cell memory.

\section{Results}

IL-7 therapy during the expansion phase does not alter the number of effector and memory CD8 Tcells during an acute LCMV infection. Here, we examined the effect of IL-7 therapy during the antigen-driven expansion phase on the primary expansion of virus-specific CD8 T cells and on the magnitude of CD8 $\mathrm{T}$ cell memory following an acute LCMV infection. Six- to eight-week-old C57BL/6 mice were infected with LCMV and treated with recombinant human IL-7 on days 1-7 after infection; mice received a daily i.p. injection of IL-7 (5 $\mu \mathrm{g} /$ mouse) or vehicle, PBS. This dose and regimen of IL-7 therapy has been shown to be efficacious and nontoxic (19). LCMV-infected C57BL/6 mice recognize the following $\mathrm{D}^{\mathrm{b}}$-restricted epitopes in the viral nucleoprotein (NP) and glycoprotein (GP): NP396-404 (NP396), GP33-41 (GP33), and GP276-285 (GP276). On day 8 after infection (peak of LCMV-specific clonal expansion), the number of epitope-specific CD8 $\mathrm{T}$ cells in the spleen was quantitated by intracellular cytokine staining (Figure 1, A and B). The absolute numbers of epitopespecific CD8 T cells in the spleens of individual IL-7-treated and control mice on day 8 after infection are shown in Figure 1A. High numbers of LCMV-specific CD8 T cells were detected in both IL-7and PBS-treated mice. Although, the spleens of IL-7-treated mice contained slightly more LCMV-specific CD8 T cells than did those of PBS-treated mice, the differences were not statistically significant $(P<0.05)$. We also determined whether exposure of CD8 T cells to supraphysiological levels of IL-7 during the expansion phase affected the number of memory CD8 T cells (Figure 1, C and D). The data in Figure 1C show that the number of LCMV-specific memory CD8 T cells (day 41 after infection) in the spleens of PBSand IL-7-treated mice were not significantly different $(P<0.05)$. Thus, exposure of CD8 T cells, in vivo, to exogenous IL-7 during the phase of primary antigen-driven proliferation did not have a detectable effect on the magnitude of expansion and contraction of effector CD8 T cells or on the size of CD8 T cell memory.

IL-7 treatment during the early contraction phase attenuates clonal downsizing of effector CD8 T cells and increases the size of CD8 T cell memory to $L C M V$. Following the expansion phase, $90-95 \%$ of the expanded LCMV-specific effector CD8 T cells are eliminated between days 8 and 30 after infection, which constitutes the contraction phase. The size of CD8 T cell memory is governed by the magnitudes of clonal expansion and/or clonal contraction of antigen-specific CD8 T cells during a $T$ cell response. Because the generation and maintenance of CD8 T cell memory is dependent on IL-7 $(5,7)$, it was of interest to determine whether clonal downsizing of effector CD8 T cells might be due to lack of sufficient IL-7. Therefore, we explored whether IL-7 supplementation during the contraction phase would inhibit loss of virus-specific CD8 T cells and enhance the size of CD8 T cell memory. Following LCMV infection, mice were treated with IL-7 or PBS daily between days 7 and 14 after infection during the contraction phase of the CD8 T cell response. On day 15 after infection, LCMV-specific CD8 T cells were enumerated in the spleens of PBS- and IL-7-treated mice by intracellular staining for IFN- $\gamma$. As illustrated in Figure 2A, spleens of IL-7-treated mice contained a significantly $(P<0.05 ; \sim 3$-fold) greater number of LCMV-specific CD8 T cells on day 15 after infection than did those of control PBStreated mice; analyses of lymph nodes and liver also showed similar differences between PBS- and IL-7-treated mice (data not shown). Notably, relative to the peak number of LCMV-specific CD8 T cells present in spleens of PBS-treated mice on day 8 after infection, the magnitude of the ensuing contraction of epitope-specific CD8 T cells (between days 7 and 14 after infection) in IL-7-treated mice was reduced by $50-60 \%$ as compared with PBS-treated mice. Collectively, 

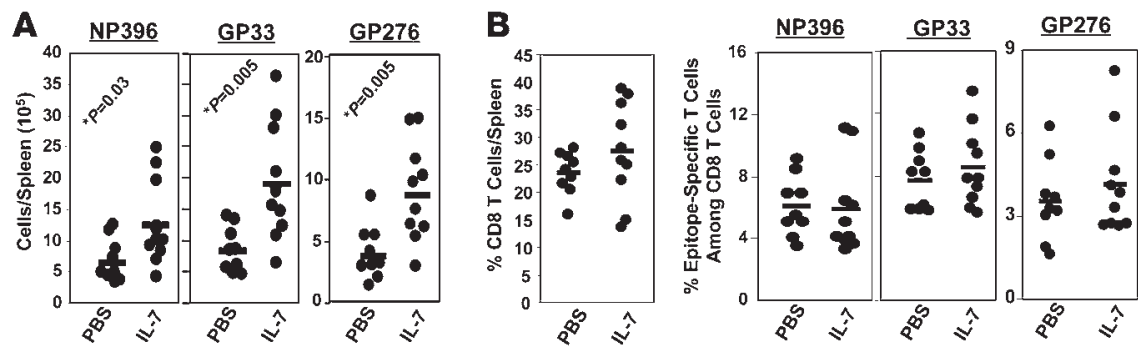

Figure 2

IL-7 treatment during the contraction phase attenuates clonal downsizing of antigen-specific CD8 T cells. C57BL/6 mice were infected with LCMV and treated daily with IL-7 or PBS between days 7 and 14 after infection. On day 15 after infection, CD8 T cells in the spleen specific to the indicated LCMV epitopes (NP396, GP33, and GP276) were quantitated by intracellular staining for IFN- $\gamma$. Following staining, cells were analyzed by flow cytometry to determine the percentages of CD8 T cells and epitope-specific IFN- $\gamma$-producing CD8 T cells in spleens of PBS- and IL-7-treated mice. (A) Total number of epitope-specific IFN- $\gamma$-producing CD8 T cells in the spleens of PBS- and IL-7-treated mice. (B) Percentages of CD8 T cells/spleen or percentages of epitope-specific CD8 T cells among total CD8 T cells in the spleens of IL-7-and PBS-treated mice. Note that each symbol in $\mathbf{A}$ and $\mathbf{B}$ represents data from the analysis of individual mice.

these data indicated that IL-7 therapy reduced clonal downsizing of LCMV-specific CD8 T cells during the contraction phase of the primary anti-viral CD8 $\mathrm{T}$ cell response. Next, we sought to decipher whether IL-7-induced effects are linked to a selective increase in the number of LCMV-specific CD8 T cells and/or a global increase in CD8 T cells in spleen. To this end, we compared percentages of CD8 $\mathrm{T}$ cells per spleen and percentages of epitope-specific cells among total CD8 T cells between PBS- and IL-7-treated groups (Figure 2B). The increased number of LCMV-specific CD8 T cells in spleens of individual IL-7-treated mice showed a strong correlation (correlation coefficient: 0.921 ) with the percentage of LCMV-specific cells among total CD8 $\mathrm{T}$ cells, but not with the percentage of CD8 T cells per spleen. These analyses suggested that IL-7 treatment expanded the number of LCMV-specific CD8 T cells within the CD8 T cell compartment. Additionally, IL-7 treatment induced an approximate 2 -fold increase in the total number of splenocytes (including CD8 $\mathrm{T}$ cells), which in turn further augmented the net increase in the number of LCMV-specific CD8 T cells. In summary, IL-7 treatmentinduced alterations in the number of LCMV-specific CD8 T cells on day 15 after infection are linked to an increase in the frequencies of virus-specific CD8 $\mathrm{T}$ cells among total CD8 T cells and with an overall increase in the total number of CD8 T cells in the spleen.

It has been reported that IL-7 might promote T cell survival by inducing the expression of the antiapoptotic molecules $\mathrm{Bcl}-2$ and $\mathrm{Bcl}-\mathrm{x}_{\mathrm{L}}(6,8)$ or serine protease inhibitor 2A (20). Moreover, over expression of $\mathrm{Bcl}-2$ can rescue $\mathrm{T}$ cell development in IL-7R $\alpha$ chaindeficient mice (21). We therefore investigated whether inhibition of CD8 $\mathrm{T}$ cell contraction due to IL-7 treatment was associated with increased cellular Bcl-2 or Bcl- $\mathrm{x}_{\mathrm{L}}$ in LCMV-specific CD8 T cells in vivo. Mice were infected with LCMV and treated daily with IL-7 between days 7 and 14 after infection. On day 15 after infection, splenocytes were stained with anti-CD8, MHC I tetramers, anti-Bcl-2, and anti-Bcl- $\mathrm{x}_{\mathrm{L}}$. As shown in Figure 3A, the levels of Bcl-2 (mean fluorescence intensity) in LCMV-specific CD8 T cells from IL-7treated mice were significantly $(P<0.05)$ higher than those from PBS-treated mice. However, IL-7 treatment did not affect the expression of Bcl- $\mathrm{x}_{\mathrm{L}}$ in LCMV-specific CD8 T cells, which is consistent with a recent report that $\mathrm{Bcl}-\mathrm{x}_{\mathrm{L}}$ plays a minor role in memory $\mathrm{T}$ cell genperipheral CD4 and CD8 T cells (26). To determine the effect of IL-7 treatment between days 7 and 14 after infection on proliferation of LCMV-specific CD8 ${ }^{+} \mathrm{T}$ cells, we measured BrdU incorporation in vivo by flow cytometry. The data in Figure 3C show that IL-7 treatment increased the relative proportions of BrdU ${ }^{+v e} \mathrm{LCMV}$-specific $\mathrm{CD} 8 \mathrm{~T}$ cells in spleen, lymph nodes, and liver compared with those in PBS-treated mice. Collectively, the data in Figure 3, B and C, suggest that IL-7-induced inhibition of clonal downsizing of LCMV-specific effector CD8 T cells was associated with increased cellular proliferation but not with the suppression of cellular apoptosis.

Next, we examined whether IL-7 treatment during the contraction phase affected the number of memory CD8 T cells. As above, mice were treated with IL-7 or PBS between days 7 and 14 after infection, and the number of LCMV-specific memory CD8 T cells was quantitated in the spleen on day 43 after infection. The number of memory CD8 T cells specific to the LCMV epitopes GP33 and GP276, but not specific to NP396, were significantly $(P<0.05 ;>200 \%)$ higher in IL-7-treated mice than in controls (Figure 4A). Similarly, IL-7 treatment augmented the number of LCMV-specific memory CD8 $\mathrm{T}$ cells in lymph nodes, bone marrow, and liver (data not shown). By contrast with the inhibitory effects of IL-7 on CD8 T cell contraction between days 7 and 15 after infection, the losses of LCMV-specific CD8 T cells between days 15 and 43 after infection in PBS- and IL-7-treated mice were comparable in magnitude. Thus, the increase in the number of LCMV-specific memory CD8 T cells in IL-7-treated mice corresponded with the inhibition of contraction between days 7 and 15 after infection. Nevertheless, the fold contraction for GP33-specific CD8 $T$ cells between days 8 and 43 after infection was calculated to be 69.9- and 27.6-fold in PBS- and IL-7-treated mice, respectively. As shown in Figure 4B, the percentages of both CD8 T cells per spleen and of GP33-specific CD8 T cells (but not NP396specific CD8 T cells) in CD8 T cells were significantly greater in IL-7treated mice than in PBS-treated mice. Although not statistically significant, the average percentage of GP276-specific cells in total CD8 T cells was greater in IL-7-treated mice than in PBS-treated mice. Additionally, the frequencies of GP33- and GP276-specific CD8 T cells in splenocytes were approximately 2 -fold greater in IL-7treated mice than in PBS-treated mice (data not shown). Notably, 
A
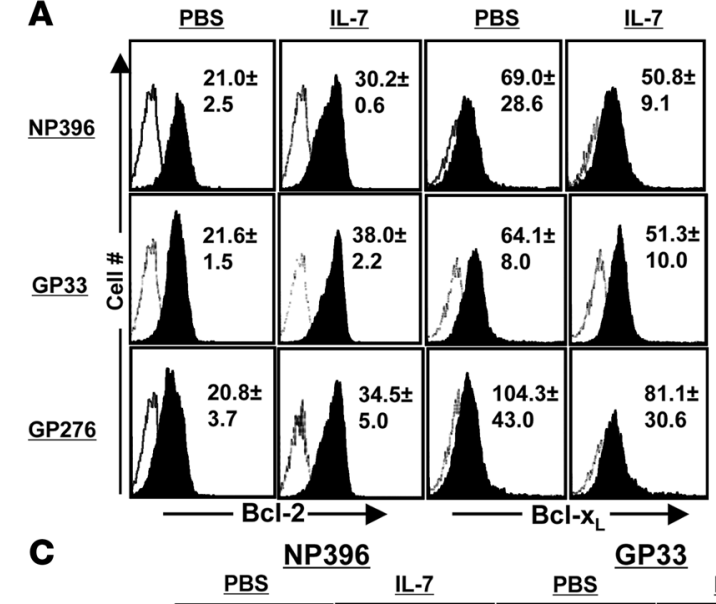

B

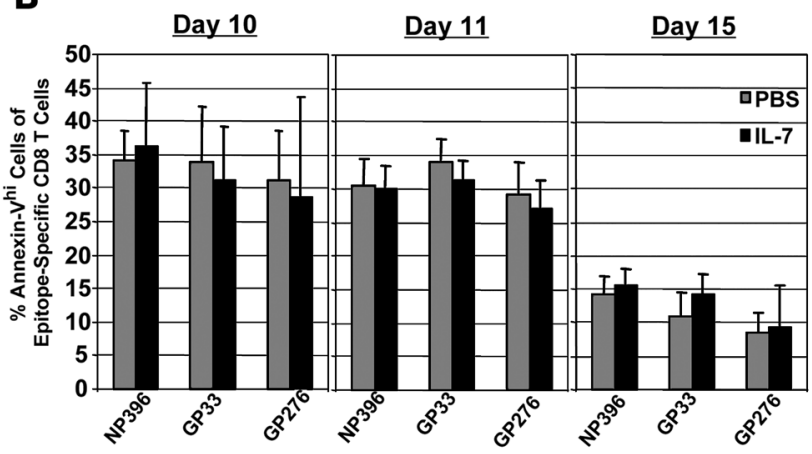

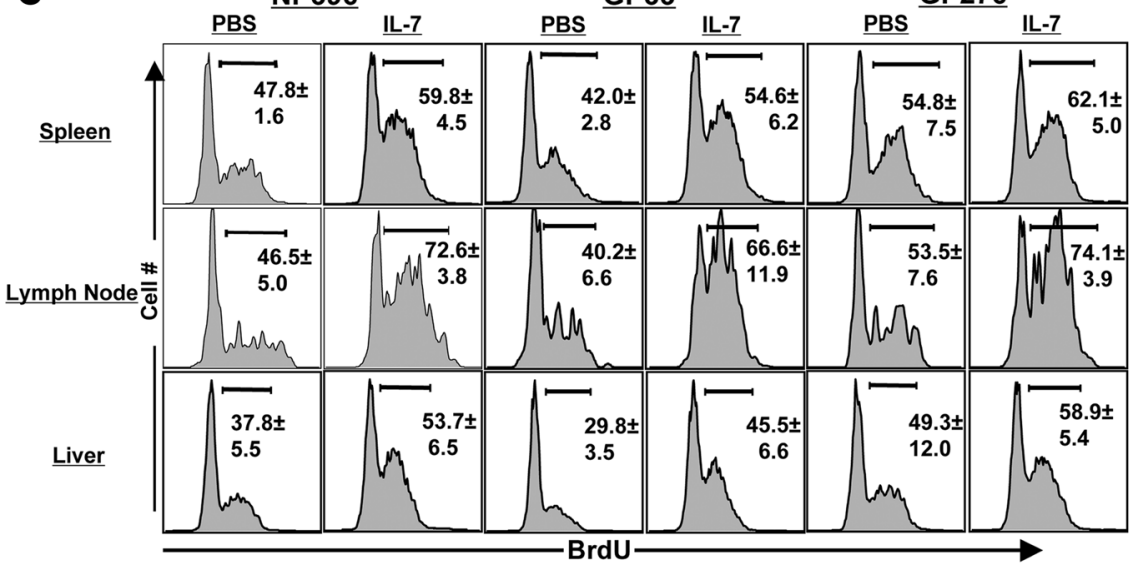

Figure 3

Effect of IL-7 treatment during the contraction phase on cellular apoptosis and proliferation. C57BL/6 mice were infected with LCMV and treated daily with IL-7 or PBS between days 7 and 14 after infection. (A) Bcl-2 and Bcl- $x_{L}$ expression in LCMV-specific CD8 T cells. On day 15 after infection, intracellular levels of $\mathrm{Bcl}-2$ and $\mathrm{Bcl}-\mathrm{x}_{\mathrm{L}}$ in epitope-specific CD8 T cells from PBS and IL-7-treated mice were determined by flow cytometry. The histograms are gated on tetramer-binding CD8 T cells and the numbers are the mean fluorescence intensity of staining for $\mathrm{Bcl}-2$ or $\mathrm{Bcl}-\mathrm{x}_{\mathrm{L}} \pm \mathrm{SD}$. (B) On the indicated days after LCMV infection, the number of proapoptotic epitope-specific annexin Vhi CD8 T cells in the spleen was assessed directly ex vivo using flow cytometry. (C) Proliferation of LCMV-specific CD8 T cells in PBS- and IL-7-treated mice. Mice were treated with BrdU between days 7 and 14 after infection, and in vivo BrdU incorporation by epitope-specific CD8 T cells was determined by flow cytometry. The histograms are gated on tetramer-binding CD8 T cells, and the numbers are the percentages of BrdU+ve cells among epitope-specific CD8 T cells \pm SD. The data in $\mathbf{A}-\mathbf{C}$ were derived from 4 to 5 mice/group.

increases in the percentages of antigen-specific CD8 T cells in total CD8 T cells (Figure 4B) for GP33 and GP276 epitopes induced by IL-7 therapy corresponded with (respective correlation coefficients of 0.87 and 0.95 ) an enhancement in the absolute numbers of CD8 $\mathrm{T}$ cells per spleen only for the respective epitopes (Figure 4A). Collectively, these data suggest that IL-7 treatment during the contraction phase enhanced the number of memory CD8 T cells that are specific to only certain epitopes, and, by extension, the augmented CD8 $\mathrm{T}$ cell memory in IL-7-treated mice could not be explained by an increase in total CD8 T cells. At 100 days after infection, although the numbers of LCMV-specific memory CD8 T cells in spleen were comparable in both groups of mice, the frequencies of GP33-specific CD8 T cells in the bone marrow cells of IL-7- and PBS-treated mice were $0.13 \% \pm 0.02 \%$ and $0.22 \% \pm 0.08 \%$, respectively. Thus, IL-7 treatment during contraction enhanced LCMV-specific memory CD8 T cells in both lymphoid and nonlymphoid tissues in the short term (up to day 43 after infection), but long-term enhancement in the number of memory CD8 $\mathrm{T}$ cells was restricted to bone marrow - a repository for central memory cells (27).
We also compared the cell-surface phenotype of memory CD8 T cells from control and IL-7-treated mice. Interestingly, we found that the fraction of CD127hi (IL-7R $\alpha$ ) cells of GP33-specific CD8 T cells (not of NP396-specific CD8 T cells) was higher in the spleens of IL-7-treated mice $(\sim 81 \%)$ than in the spleens of PBS-treated mice ( $69 \%$ ) (Figure $4 \mathrm{C})$; in the same cells, levels of both CD122 and CD44 were not affected by IL-7 treatment (data not shown). Next, we asked whether IL-7 treatment during the contraction phase affected the cytokine-producing ability of LCMV-specific memory CD8 T cells. On day 43 after infection, we assessed the ability of LCMV-specific memory CD8 T cells to produce IFN- $\gamma$, TNF- $\alpha$, and IL- 2 ex vivo in response to antigenic stimulation. It is well established that all LCMV-specific memory CD8 T cells can be stimulated to produce IFN- $\gamma$, but only a subpopulation of these memory cells also produces IL-2 $(28,29)$. In both IL-7-treated and PBS-treated mice, most of the IFN- $\gamma$-producing CD8 T cells also produced TNF- $\alpha$ (Figure 4D). Likewise, as shown in Figure 4D, the fraction of IL-2-producing CD8 T cells among LCMV-specific CD8 T cells was comparable in IL-7- and PBS-treated mice. 


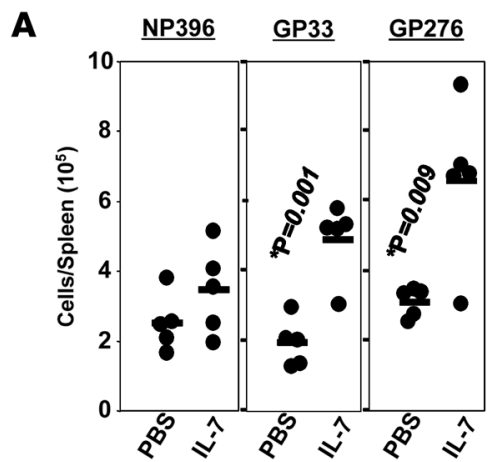

C

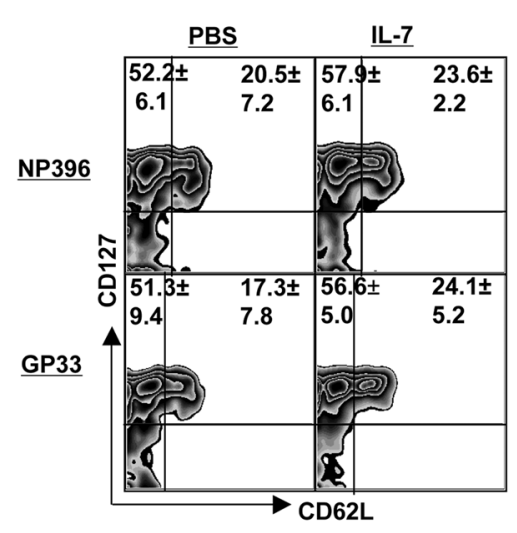

B
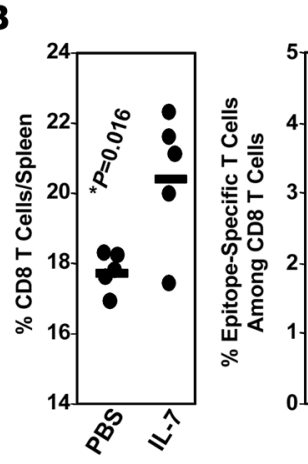

NP39

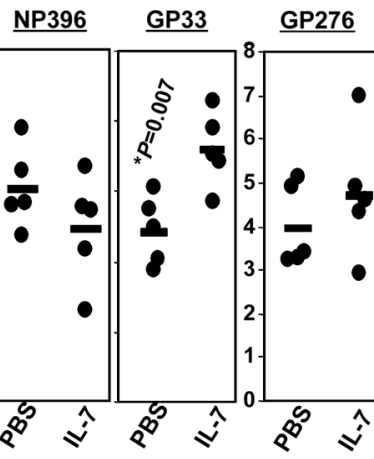

D

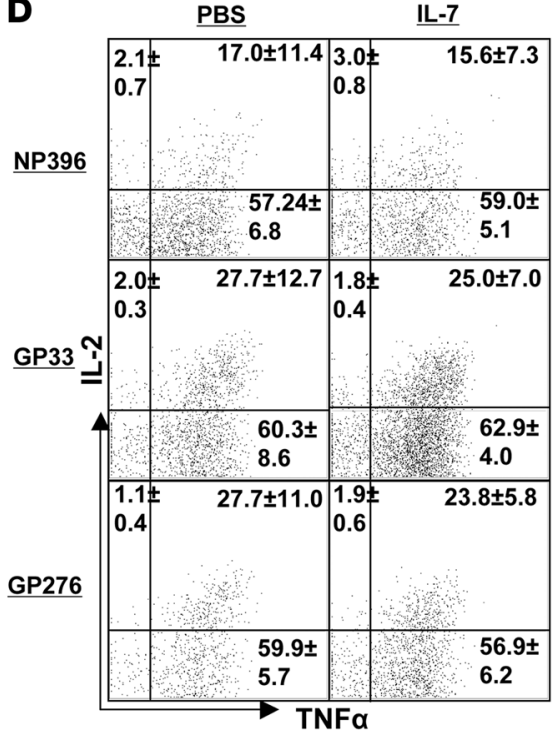

Figure 4

IL-7 treatment during contraction enhances CD8 $T$ cell memory to LCMV. LCMV-infected C57BL/6 mice were treated daily with IL-7 or PBS between days 7 and 14 after infection. (A and B). On day 43 after infection, the number of epitopespecific CD8 T cells in the spleen was enumerated by staining with MHC I tetramers. Each data point represents an individual mouse. (C) Cell surface phenotype of memory CD8 T cells. On day 43 after infection, splenocytes were stained with anti-CD8, MHC I tetramers, anti-CD127, and anti-CD62L. The fluorescence activated cell sorting plots are gated on tetramer-binding CD8 T cells, and the numbers are the percentages of cells among epitope-specific cells in the respective quadrants \pm SD. (D) Cytokine production by memory CD8 T cells. The ability of LCMV-specific memory CD8 T cells to produce IFN- $\gamma$, TNF, and IL-2 was assessed by intracellular cytokine staining; dot plots are gated on IFN- $\gamma$-producing CD8 T cells, and the numbers represent cells in each quadrant as a percentage \pm SD among IFN- $\gamma$-producing epitope-specific CD8 T cells. The data in $\mathbf{C}$ and $\mathbf{D}$ were derived from analyses of 5 mice/group.
Therefore, IL-7 therapy during the contraction phase did not significantly affect the cytokine-producing ability of LCMV-specific memory CD8 T cells.

IL-7 therapy during the contraction phase enhances CD8 T cell memory to recombinant $V V$. Historically, $\mathrm{V}$ has been used as an effective vaccine to protect against small pox in humans. Additionally, $\mathrm{VV}$ is an excellent vaccine vector for inducing protective immunity against several infections. Here, we investigated whether IL-7 therapy during the contraction phase affected the magnitude of CD8 T cell memory after $\mathrm{VV}$ infection. We infected C57BL/6 mice with a recombinant $\mathrm{VV}$ (30) that expresses the GP of LCMV (VV-GP) and treated them daily with PBS vehicle or IL-7 between days 7 and 14 after infection, as described above. On day 15 after infection, the number of CD8 T cells specific to either the VV immunodominant epitope B8R (31) or the LCMV epitope GP33 was quantitated in the spleen by intracellular cytokine staining. As shown in Figure 5A, on day 15 after infection, the numbers of B8R- and GP33-specific CD8 $\mathrm{T}$ cells in the spleens of IL-7-treated mice were significantly higher $(P<0.05 ;>200 \%)$ than in PBS-treated mice immediately after the cessation of IL-7 therapy. As in an LCMV infection (Figure 2), on day 15 after infection, the observed increase in the number of B8R- and GP33-specific CD8 T cells in the spleens of VV-GP-infected IL-7treated mice correlated with an increased number of total splenocytes, including CD8 T cells, and was not linked to alterations in either percentages of either CD8 T cells/spleen or epitope-specific CD8 T cells among CD8 T cells (Figure 5B).
Next, we investigated the effect of IL-7 therapy during the contraction phase of the CD8 T cell response to VV-GP on the magnitude of CD8 T cell memory. The data in Figure 5C show that the number of B8R- and GP33-specific memory CD8 $\mathrm{T}$ cells was significantly greater in the spleens of IL-7-treated mice than in the spleens of PBS-treated mice. As in an LCMV infection (Figures 2 and 4), the IL-7-induced increase in the number of memory CD8 T cells in VV-GP-infected mice correlated with the inhibition of contraction between days 7 and 14 after infection but not after cessation of IL-7 treatment. Unlike on day 15 after infection (Figure 5B), the increase in the number of B8R-and GP33-specific memory CD8 $T$ cells was not related to spleen size because the total number of splenocytes in IL-7-treated mice was similar to that in PBS-treated mice. Instead, the percentages of B8R- and GP33-specific memory CD8 T cells among CD8 T cells were significantly higher in IL-7treated mice than in PBS-treated mice (Figure 5D). Moreover, the percentage of CD8 T cells per spleen in IL-7- and PBS-treated mice were not significantly different (Figure 5D). Collectively, these data show that IL-7 therapy during contraction selectively enhanced the number of epitope-specific memory CD8 T cells and did not lead to a permanent increase in total CD8 T cells. Nonetheless, the data presented in Figure 5 indicate that IL-7 treatment could be used to bolster CD8 T cell memory induced by live virus vaccines.

IL-7 therapy enhances CD 8 T cell memory and recall response to a DNA vaccine. The data in Figures 4 and 5 show that IL-7 treatment of mice during contraction phase of the CD8 $\mathrm{T}$ cell response to a replicating 
A
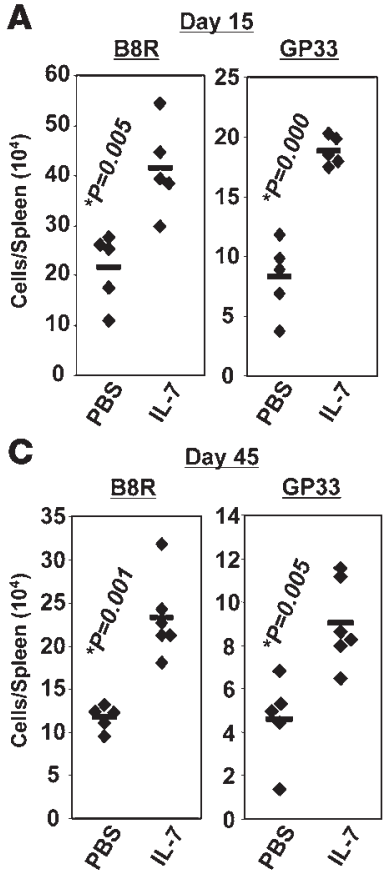

B

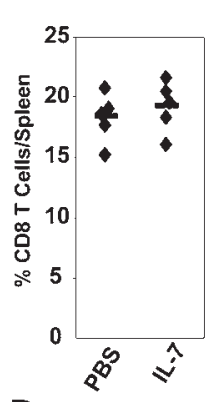

D

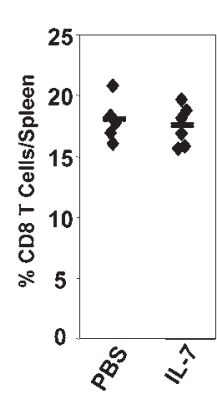

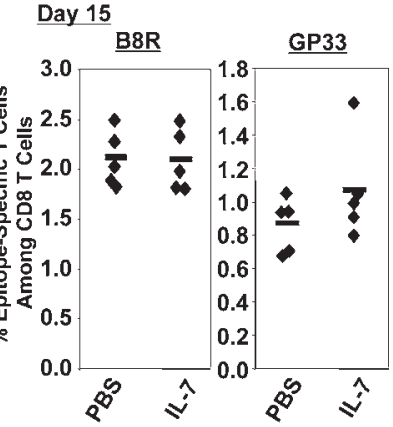

Day 45

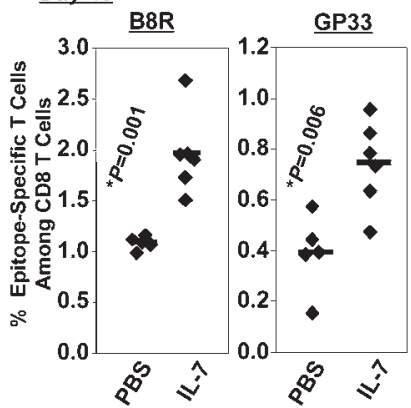

Figure 5

IL-7 treatment during contraction enhances CD8 T cell memory to VV. C57BL/6 mice were infected with recombinant VV, i.e., VV-GP that expresses the GP of LCMV. Mice were treated daily with IL-7 or PBS between days 7 and 14 after infection. On days 15 (A and B) and 45 (C and D) after infection, CD8 T cells specific to the VV epitope B8R and LCMV epitope GP33 were quantitated by intracellular staining for IFN- $\gamma$. Each symbol represents data from an individual mouse.

antigen (live virus) enhanced the number of memory CD8 $\mathrm{T}$ cells. Here, we investigated whether IL-7 can enhance CD8 T cell memory following immunization with a DNA vaccine. Groups of C57BL/6 mice were immunized with DNA plasmid pCMV-NP, which expresses the NP gene of LCMV under control of the CMV promoter; CD8 T cell response to PCMV-NP peaks on day 14 after immunization (32). Between days 14 and 21 after PCMV-NP immunization, mice were treated with IL-7 or PBS as described above. On day 35 after immunization, we quantitated NP396-specific CD8 T cells in the spleens of pCMV-NP-immunized IL-7- or PBS-treated mice. Despite the variability typically associated with DNA immunizations (33-35), the number of NP396-specific CD8 T cells was significantly greater in the spleens of IL-7-treated mice than in the spleens of PBS-treated mice (Figure 6). The enhancement in NP396-specific CD8 T cells induced by IL-7 strongly correlated (correlation coefficient: 0.939 ) with an increase in the percentage of epitope-specific cells among total CD8 T cells (Figure 6) but not with the percentage of CD8 $\mathrm{T}$ cells per spleen (Figure 6). Thus, as in a live virus infection, IL-7 therapy enhanced DNA vaccine-induced CD8 T cell memory.

Next, we determined the effect of IL-7 therapy on recall responses to DNA immunization. As above, groups of $\mathrm{C} 57 \mathrm{BL} / 6$ mice were immunized with pCMV-NP and treated with IL-7 or PBS between days 14 and 21 after immunization. On day 38 after DNA immunization, mice were challenged with LCMV-clone 13 , a virulent strain of LCMV, and recall responses were analyzed 5 days after challenge; empty vector ( $\mathrm{pCMV}$ )-immunized mice were challenged as controls. As shown in Figure 7A, the spleens of IL-7-treated pCMV-NP-immunized mice contained a substantially larger number of NP396-specif-
PBS or IL- 7 between days 7 and 14 after infection. On days 15 and 55 after infection, the number of donor TCR transgenic P14/Thy1.1 CD8 T cells in PBS- and IL-7-treated mice was quantitated by staining splenocytes with anti-CD8, anti-Thy 1.1 , and $\mathrm{D}^{\mathrm{b}} / \mathrm{GP} 33$ tetramers. On day 15 after infection, the spleens of IL-7treated mice contained $\sim 3$-fold more P14/Thy1.1 CD8 T cells than did the spleens of PBS-treated mice (Figure 8A). Similar to polyclonal responses (Figure 2A), the inflated number of P14 CD8 T cells in the spleens of IL-7-treated mice on day 15 after infection was a net result of the increase in the total number of splenocytes and frequencies of P14 CD8 T cells among splenocytes (Figure 8A). On day 55 after infection, the spleens of IL-7-treated mice contained a significantly $(P<0.002)$ greater number of $\mathrm{P} 14$ memory $\mathrm{CD} 8 \mathrm{~T}$ cells than did the spleens of PBS-treated mice (Figure 8B). The data in Figure 8B also indicate that the IL-7 treatment-induced enhancement in the number of P14 memory CD8 T cells was associated with an increase in the percentage of $\mathrm{P} 14$ cells among total CD8 T cells, but not with the percentage of CD8 $\mathrm{T}$ cells per spleen. We also calculated the effect of IL-7 therapy on the magnitude of contraction of P14 CD8 T cells. Between days 8 and 15 after infection, the number of P14 CD8 T cells in the spleens of PBS- and IL-7-treated mice decreased by 35and 11.7-fold, respectively. After cessation of IL-7 therapy, between days 15 and 55 after infection, there was a 2.6- and 4.2-fold loss of P14 CD8 T cells in PBS- and IL-7-treated mice, respectively. Thus, we confirmed that IL-7 treatment inhibited the clonal downsizing of monoclonal P14/Thy1.1 GP33-specific CD8 T cells as it did on polyclonal GP33-specific CD8 T cells (Figure 2). Additionally, these data indicate that enhancement of memory CD8 T cells induced by IL-7 is not dependent on its effects on thymopoiesis. 

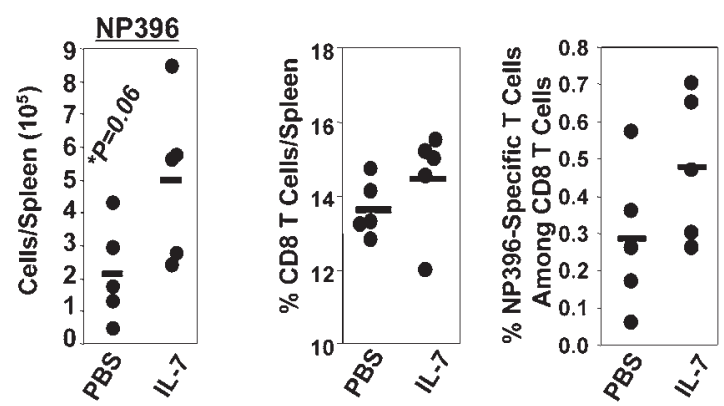

Figure 6

IL-7 treatment enhances CD8 T cell memory following DNA immunization. C57BL/6 mice were immunized with DNA plasmid pCMV-NP that expresses the NP of LCMV. Cohorts of mice were treated daily with IL-7 or PBS between days 14 and 21 after DNA immunization. On day 35 after DNA immunization, the number of NP396-specific CD8 T cells in spleen was quantitated by intracellular cytokine staining. Each symbol represents data from an individual mouse.

Effect of IL-7 therapy on the protective efficacy of virus-specific CD $8 \mathrm{~T}$ cells. The data in Figure 2A and Figure 8A show that IL-7 treatment between days 7 and 14 after infection attenuated the clonal downsizing of LCMV-specific effector CD8 T cells. Here, we investigated whether IL-7 therapy affected the ability of P14/Thy1.1 CD8 T cells to control a virulent LCMV (LCMV-clone 13) infection. As above (Figure 8), naive P14/Thy1.1 CD8 T cells were adoptively transferred into congenic Thy 1.2/C57BL/6 mice and infected with LCMV-Armstrong 53B (Arm); cohorts of adoptively transferred host mice were treated daily with PBS or IL-7 between days 7 and 14 after infection. On day 15 after infection, immediately after cessation of IL-7 treatment, $9.4 \times 10^{5}$ in vivo-activated P14/Thy1.1 CD8 $\mathrm{T}$ cells obtained from the spleens of LCMV-Arm-infected PBS- or IL-7-treated mice were adoptively transferred into naive Thy1.2/C57BL/6 mice, which were challenged with LCMV-clone 13 twenty-four hours after cell transfer. Five days after LCMVclone 13 challenge, we quantitated the secondary expansion of P14/Thy1.1 CD8 T cells in the spleen and measured infectious viral titers in the liver and lung. On LCMV-clone 13 challenge, P14/Thy1.1 CD8 T cells from IL-7-treated mice expanded almost 2-fold more than did P14 CD8 T cells from PBS-treated mice (Figure 9A). Most importantly, P14/Thy1.1 CD8 T cells from IL-7treated mice controlled LCMV-clone 13 infection more effectively than did those from PBS-treated mice; LCMV-clone 13 titers in the liver were 10-100-fold lower in mice that received P14 CD8 T cells from IL-7-treated mice than in P14 CD8 T cells from PBS-treated mice (Figure 9B). Thus, IL-7 therapy during the contraction phase improved the protective efficacy of LCMV-specific CD8 T cells.

Here, to understand how IL-7 treatment during the contraction phase increased the protective efficacy of P14 CD8 T cells, we compared the MHC I-restricted cytotoxic activity of P14 cells from IL-7or PBS-treated mice on day 15 after infection. As shown in Figure 9C, the cytotoxic activity of P14 CD8 T cells from IL-7-treated mice was greater than that from PBS-treated mice. Next, we determined whether IL-7 treatment affected the proliferative responses of P14 CD8 T cells to antigen recall by stimulating CFSE-labeled P14 CD8 $\mathrm{T}$ cells from IL-7- or PBS-treated mice with GP33 peptide in vitro. As illustrated in Figure 9D, whereas only $45 \%$ of P14 CD8 T cells from PBS-treated mice exhibited a proliferative response, $>85 \%$ of P14 CD8 T cells from IL-7-treated mice divided multiple times in response to antigenic stimulation with the GP33 peptide. Thus, IL-7 treatment during the contraction phase induced both quantitative (increased number of antigen-specific CD8 T cells) and qualitative (improved effector function and proliferative potential) alterations in LCMV-specific CD8 T cells, which might explain the enhanced protective ability of P14 CD8 T cells from IL-7-treated mice.

IL-7 treatment during the memory phase induces proliferation and enhances the number of memory CD8 T cells. To investigate whether administration of exogenous IL-7 can boost preexisting CD8 T cell memory, we treated LCMV-Arm-immune mice ( $\sim 6$ months after infection) with PBS or IL-7 daily for 11 days. As shown in Figure 10A, IL-7 treatment induced a substantive increase ( $\sim$-fold) in the number of LCMVspecific memory CD8 T cells in the spleen, lymph nodes, and liver. Apart from increasing the percentages of CD8 T cells in all peripheral lymphoid tissues including spleen, lymph nodes, and liver, IL-7 therapy also effected substantial elevation in the frequencies of LCMV-specific memory CD8 T cells among total CD8 T cells (Figure 10A). Moreover, IL-7 treatment also increased the percentages of LCMV-specific CD8 T cells among mononuclear cells by 2- to 3-fold in respective lymphoid tissues (data not shown). Collectively, these data indicate that IL-7 treatment during the memory phase not only enlarged the relative proportions of LCMV-specific memory CD8 T cells within the CD8 $\mathrm{T}$ cell compartment, but also increased the total
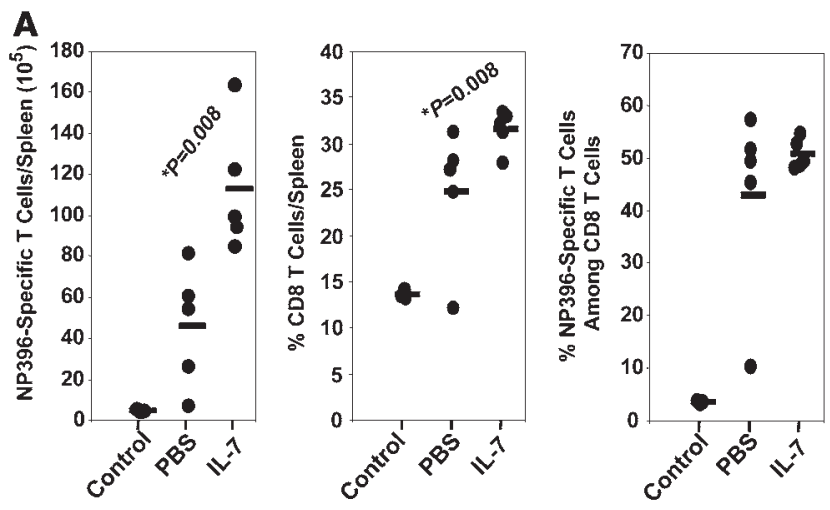

B

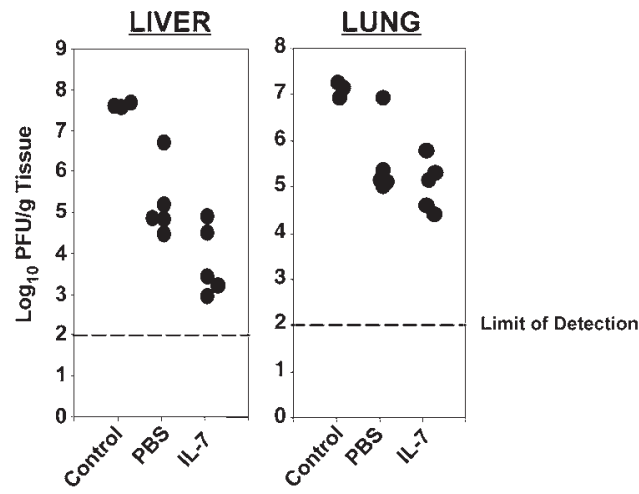

Figure 7

IL-7 treatment enhances recall CD8 T cell responses following DNA immunization. C57BL/6 mice were immunized with empty vector $\mathrm{pCMV}$ (control) or pCMV-NP. pCMV-NP-immunized mice were treated with IL-7 or PBS between days 14 and 21 after DNA injection and then challenged with LCMV-clone 13 on day 38 after DNA immunization. On day 5 after the LCMV-clone 13 challenge, the number of NP396specific CD8 T cells in spleen was quantitated by intracellular cytokine staining $(\mathbf{A})$, and LCMV titers in the tissues were assessed by plaque assay (B). Each symbol represents data from an individual mouse. 
A

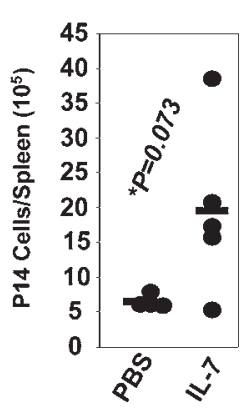

B

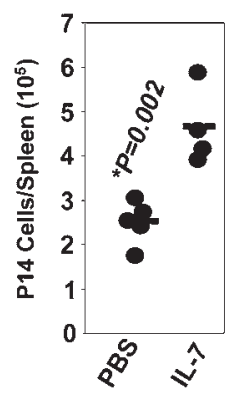

Day 15
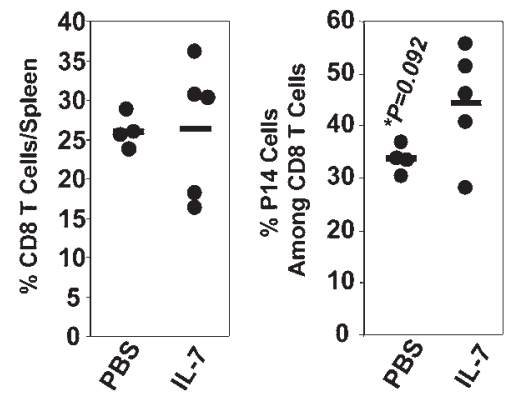

Day 55

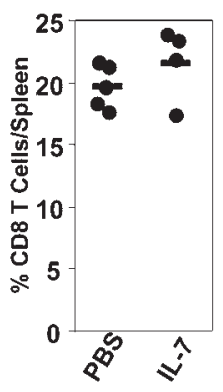

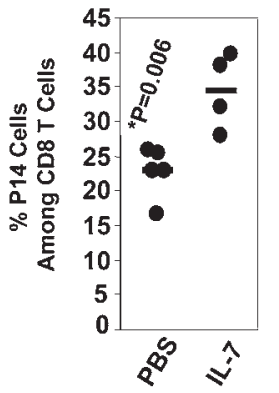

CD8 T cell pool in peripheral lymphoid tissues. This enhancement in the number of LCMV-specific memory CD8 T cells induced by the 11-day IL-7 regimen was associated with increased cell proliferation, as shown in Figure 10B. Notably, both in the spleen and lymph nodes, IL-7 treatment led to a small but significant $(P<0.05)$ increase in the relative proportions of CD62L hi central memory CD8 T cells and a corresponding reduction in the percentages of CD62 $\mathrm{L}^{\text {lo }}$ effector memory CD8 T cells (Figure 10C). Next, we examined whether enhancement in the number of memory CD8 T cells in LCMVimmune mice was transient or was stably increased by IL-7 therapy. Eighty days after the last IL-7 injection, the numbers of LCMV-specific memory CD8 T cells in the spleen, lymph nodes, liver, and bone marrow of IL-7-treated mice were comparable with those in control PBS-treated mice (Figure 10D). Thus, in LCMV-immune mice, an 11-day course of daily IL-7 treatment was able, by itself, to effect a substantial increase in the number of LCMV-specific memory CD8 T cells in both lymphoid and nonlymphoid organs. However, this IL-7induced expansion of fully differentiated memory CD8 T cells was not a permanent change in the memory compartment.

Effect of IL-7 administration during the contraction phase of a secondary response. Next, we determined the effect on $\mathrm{CD} 8 \mathrm{~T}$ cell memory when IL-7 was administered during the contraction phase of a secondary $\mathrm{T}$ cell response. LCMV-Arm-immune mice were challenged with recombinant Listeria monocytogenes that expresses the GP33 epitope of LCMV (LM-GP33) and treated with IL-7 or the vehicle PBS daily for 10 days during the contraction phase (days 5-15 after LM-GP33 challenge) of the secondary CD8 T cell response. On day 34 after LM-GP33 challenge, we quantitated the number of GP33-specific CD8 T cells in the spleens of IL-7- and PBS-treated mice (Figure 11A). The spleens of IL-7-treated mice contained a significantly greater $(P=0.05)$ number of GP33-specific CD8 T cells than did the spleens of PBS-treated mice. In contrast, the total number of NP396- and GP276-specific memory CD8 T cells in the spleens of IL-7-treated mice was comparable with that in PBS-treated mice.

\section{Figure 8}

IL-7 treatment attenuates clonal downsizing of TCR transgenic CD8 T cells and enhances CD8 T cell memory. Naive Thy1.1+ve P14 CD8 T cells were adoptively transferred into congenic Thy $1.2 / \mathrm{C} 57 \mathrm{BL} / 6$ mice and infected with LCMV. Between days 7 and 14 after infection, mice received daily injections of IL-7 or PBS. On days 15 (A) and 55 (B) after infection, the number of TCR transgenic P14 CD8 T cells in the spleen was quantitated by staining splenocytes with anti-Thy1.1, D $/ G P 33$ tetramers, and anti-CD8. Each symbol point represents data from an individual mouse.

The percentages of CD8 T cells/spleen or GP33-specific CD8 T cells among total CD8 $\mathrm{T}$ cells were not significantly affected by IL-7 therapy (Figure 11B). Thus, similar to a primary response (Figure 4), IL-7 therapy during the contraction phase of a recall CD8 $\mathrm{T}$ cell response can increase the magnitude of secondary memory CD8 T cells.

\section{Discussion}

It is well established that IL-7 is a uniquely nonredundant cytokine that regulates the cell fate decisions of immature $T$ cells during thymic development and of mature $\mathrm{T}$ cells in the periphery (4). Therefore, IL-7 has been considered a master regulator of T cell homeostasis, and clinical trials are underway to evaluate the immunotherapeutic benefits of IL-7 therapy in humans (11-14). However, our understanding of the factors that influence the immunological effects of exogenous IL-7 administration is sorely incomplete. In this study, we performed a systematic, detailed analysis of the effect of timing of IL-7 therapy during the immune response on the quantity and quality of CD8 T cell memory. We showed that the ability of IL-7 therapy to enhance CD8 T cell memory depends on the timing of treatment during the $\mathrm{T}$ cell response and the antigenic specificity of the responding CD8 $\mathrm{T}$ cells. These findings have clear implications for the use of IL-7 to boost immune memory during vaccinations.

One therapeutic application in cytokine therapy is to administer IL-7 as an adjuvant at the time of prophylactic vaccination. Previous studies have shown that daily IL-7 administration extended over 28 days, to include both expansion and contraction phases of the $\mathrm{T}$ cell response, increased the number of antigen-specific CD8 T cells measured on day 29 (17). In contrast with IL-7 administration for 28 consecutive days, which might have practical constraints, we investigated the possibility of reducing the duration of IL-7 therapy to enhance CD8 T cell responses. More importantly, the effect of exogenous IL-7 treatment limited to the duration of expansion, contraction, or memory phase on CD8 T cell memory had not been explored. Our studies showed that IL-7 treatment restricted to the expansion phase (days 0-7 after infection) of the anti-LCMV T cell response did not reproducibly affect either the magnitude of primary expansion or the size of CD8 T cell memory. These findings were not exclusive to acute LCMV infection because IL-7 treatment during the primary response to L. monocytogenes infection also yielded similar results (data not shown). Nonetheless, these data suggest that a paucity of IL-7 would not be a constraint for clonal expansion of CD8 T cells during a primary response to LCMV. These studies also indicated that exposure of CD8 T cells to supra-physiological levels of IL-7 during the antigen-driven expansion phase did not affect the programmed contraction of activated CD8 T cells and survival of memory T cells. Among other factors, TCR signaling leads to profound downregulation of IL-7R $\alpha$ expression on T cells $(5,37)$. Thus, during the period of antigen-driven expansion, CD8 
A
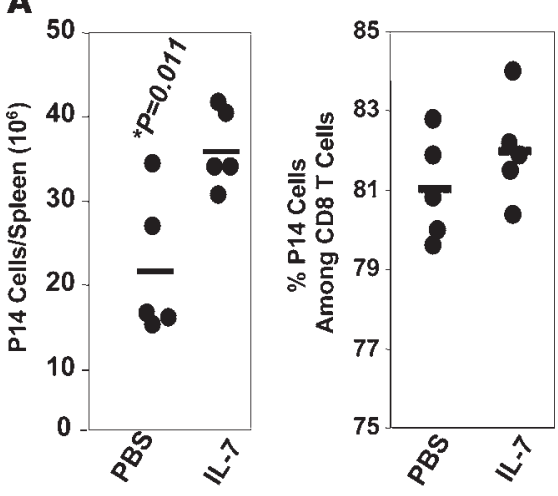

C

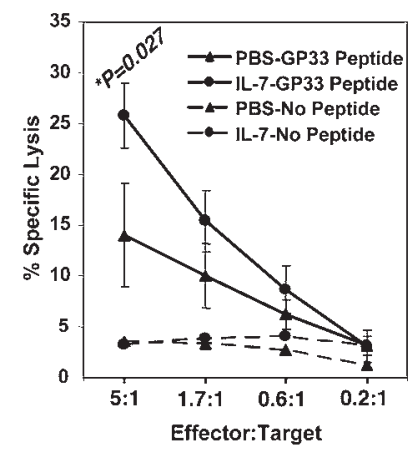

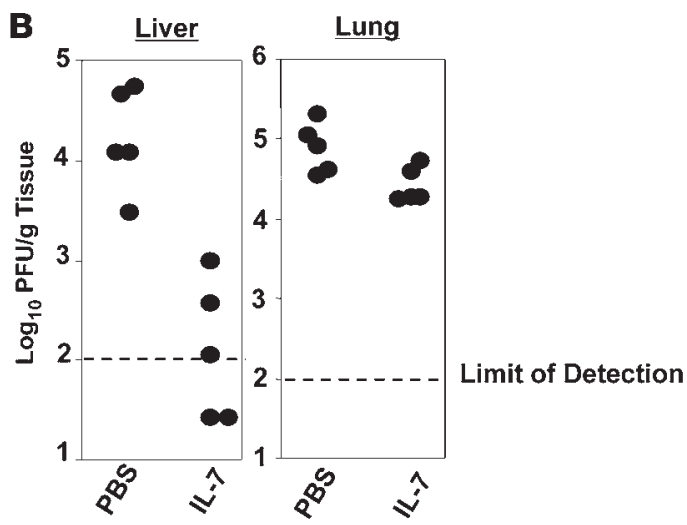

D

PBS

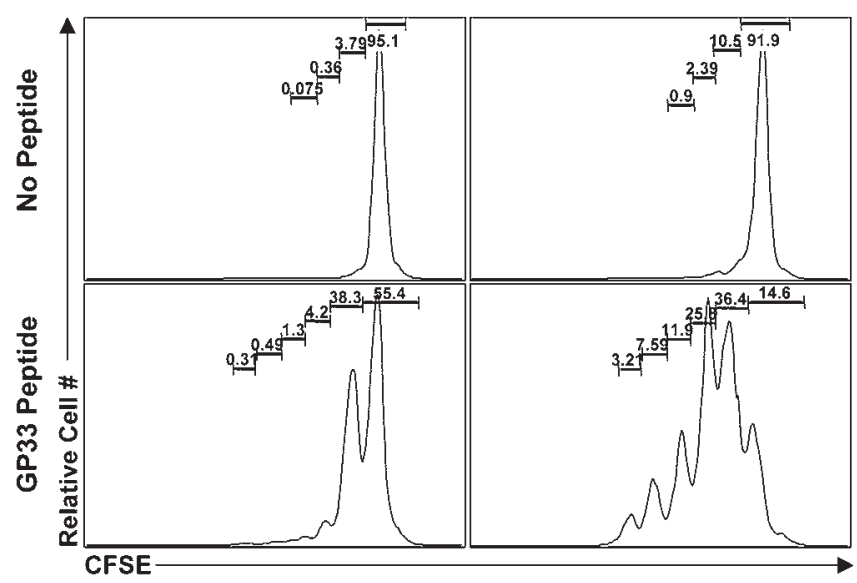

Figure 9

Protective efficacy of P14 CD8 T cells from IL-7 treated mice. Naive Thy1.1+ve or Ly5.1+ve P14 CD8 T cells were adoptively transferred into congenic Thy1.2/C57BL/6 mice and infected with LCMV-Arm. Between days 7 and 14 after infection, mice received daily injections of IL-7 or PBS. (A and B) Secondary expansion and protective immunity of P14 CD8 T cells from IL-7-treated mice. On day 15 after infection, T cells were purified from the spleens of LCMV-Arm-infected PBS- and IL-7-treated mice, and equal numbers of in vivo-activated P14 CD8 T cells were adoptively transferred into Thy1.2/C57BL/6 mice. Recipient mice were challenged with LCMV-clone 13 one day after cell transfer; 5 days after LCMV-clone 13 challenge, the secondary expansion of P14 CD8 T cells in the spleen (A) and viral titers in the lung and liver (B) were quantitated. (C) Cytotoxic activity of P14 CD8 T cells from IL-7-treated mice. On day 15 after primary LCMV infection, the number of P14 CD8 T cells from the spleen were normalized between samples and tested for cytotoxic activity at the indicated effector/target ratios using GP33-pulsed (GP33 peptide) or unpulsed (no peptide) MC57 target cells directly ex vivo. (D) Antigen-induced proliferation of P14 CD8 T cells from IL-7-treated mice. On day 15 after infection, CFSE-labeled splenocytes were stimulated in vitro with GP33 peptide for 60 hours. The histograms show CFSE fluorescence in gated P14 CD8 T cells. Note the cell division-induced dilution of CFSE in P14 CD8 T cells from IL-7-treated mice.

T cells might be refractory to IL-7 because of the downregulation of cell-surface IL-7R $\alpha$. Nonetheless, our studies showed that administration of exogenous IL-7 during the antigen-driven expansion phase of either a primary or a secondary challenge (data not shown) does not reliably enhance CD8 $\mathrm{T}$ cell immunity.

On the other hand, in the present study, treatment of mice with IL-7 during the early contraction phase (days 7-14 after infection) significantly attenuated clonal downsizing of effector cells and subsequently enhanced the number of memory CD8 T cells. Consistent with a recently published report (38), administration of a supraphysiological dose of IL-7 in the present study only attenuated and did not abrogate the contraction of activated CD8 T cells, which suggests that a paucity of IL-7 might not be solely responsible for clonal downsizing. What is the mechanism underlying the enhancement of memory CD8 T cells by this timing of IL-7 treatment? IL-7 is known to promote T cell survival by inducing the expression of antiapoptotic Bcl-2 or MCL-1 or serine protease inhibitor $2 \mathrm{~A}$ and/or by suppressing the proapoptotic activity of $\operatorname{BIM}(6,8,20,39-41)$; overexpression of $\mathrm{Bcl}-2$ or genetic deficiency of BIM could rescue $\mathrm{T}$ cell development in IL-7R $\alpha$ chain-deficient mice $(21,39)$. In addition to its antiapoptotic effects, high doses of IL-7 have been reported to induce cellular proliferation by downregulating the activity of the CDK inhibitor $p 27^{K i p} 1$ (25). In our studies, IL-7 therapy induced upregulation of antiapoptotic Bcl-2, but did not significantly affect the apoptosis of LCMV-specific CD8 T cells. In contrast, IL-7 therapy down-

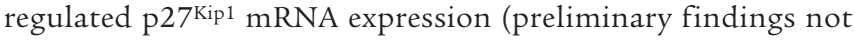
shown) and increased the proliferation of LCMV-specific CD8 T cells in vivo. Collectively, our findings suggest that administration of supra-physiological doses of IL-7 might enhance the magnitude of CD8 $\mathrm{T}$ cell memory by inducing cellular proliferation and not by preventing apoptosis. 
A
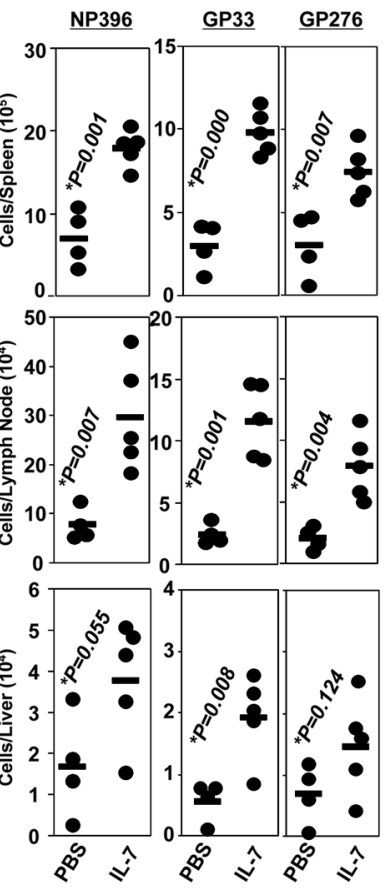

NP396

Liver

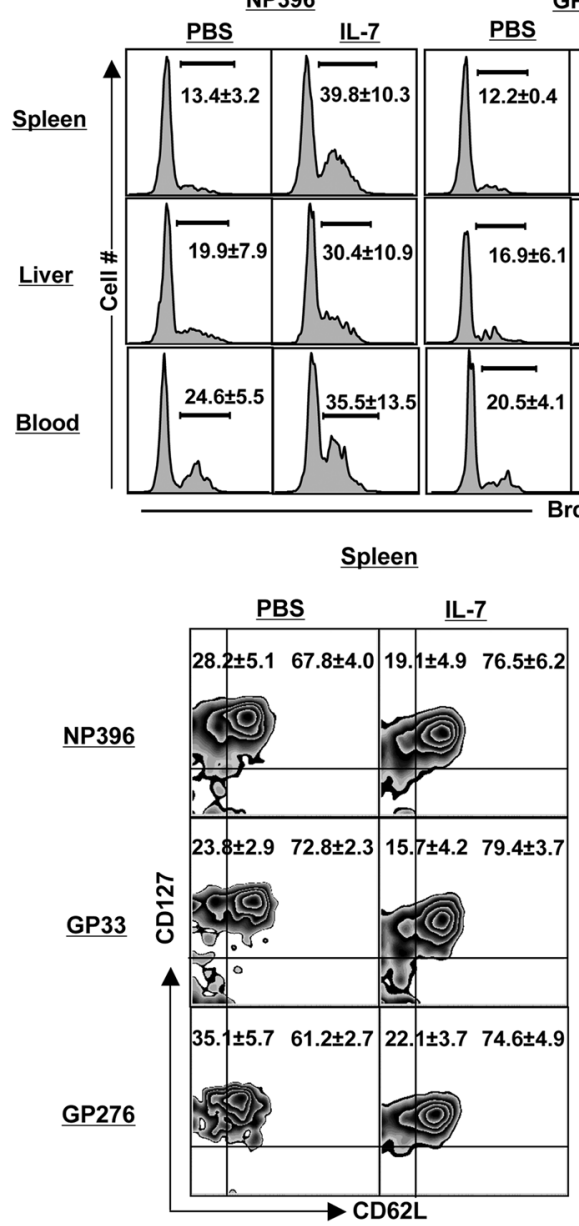

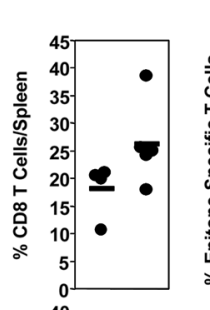
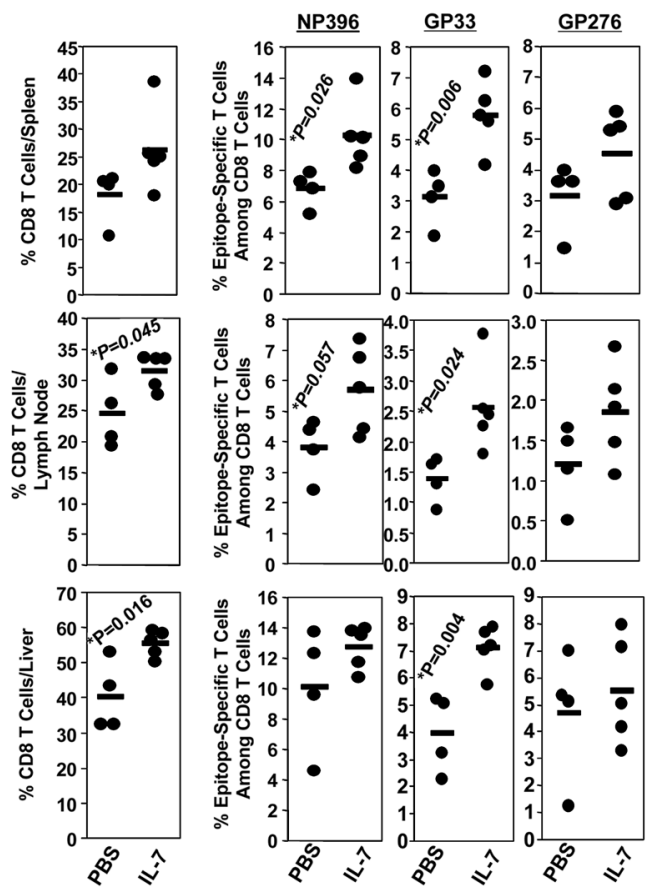

GP276 IL-7 IL-7
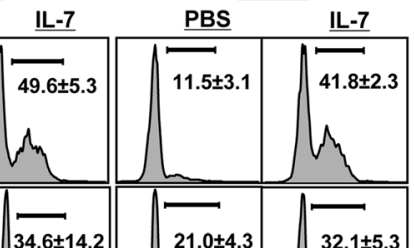

$21.0 \pm 4.3$
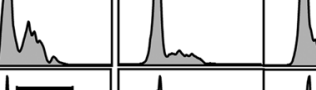

(1)

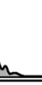

$-37.6 \pm 9.2$ m
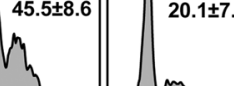

\section{D}
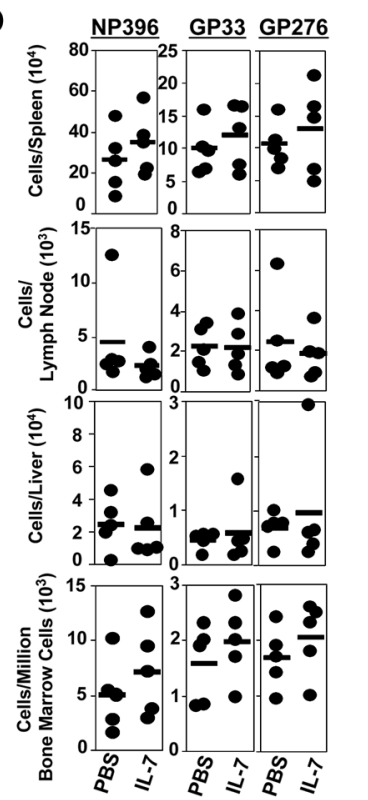

Figure 10

IL-7 treatment of mice during the memory phase leads to a transient increase in the number of LCMV-specific CD8 T cells. Approximately 6 months after LCMV infection, cohorts of LCMV-immune mice were treated daily with IL-7 or PBS for 11 days. On days 12 (A) and 90 (D) after IL-7 treatment, LCMV-specific CD8 T cells in the spleen, lymph nodes, and liver were quantitated by staining with anti-CD8 and $\mathrm{MHC}$ I tetramers. Each symbol represents total epitope-specific CD8 T cells in individual mice. (B) IL-7 treatment induces proliferation of LCMVspecific memory CD8 T cells. LCMVimmune mice were administered BrdU in drinking water during IL-7 treatment (days 0 to day 7); BrdU incorporation by epitope-specific CD8 T cells in the spleen was determined by flow cytometry on day 8 . The histograms are gated on tetramer-binding CD8 T cells, and the numbers are the percentages of $\mathrm{BrdU}^{+\mathrm{ve}}$ cells among epitope-specific CD8 T cells \pm SD $(n=5)$ (C) Cellsurface phenotype of LCMV-specific memory CD8 T cells in IL-7-treated mice. On day 12 after the initiation of IL-7 treatment, cells were stained with anti-CD8, anti-CD127, anti-CD62L, and $\mathrm{MHC}$ I tetramers; fluorescence activated cell sorting plots are gated on tetramer-binding CD8 T cells, and the numbers are the percentages of cells in each quadrant of total epitope-specific $\mathrm{CD} 8 \mathrm{~T}$ cells $\pm \mathrm{SD}(n=5)$. 
A
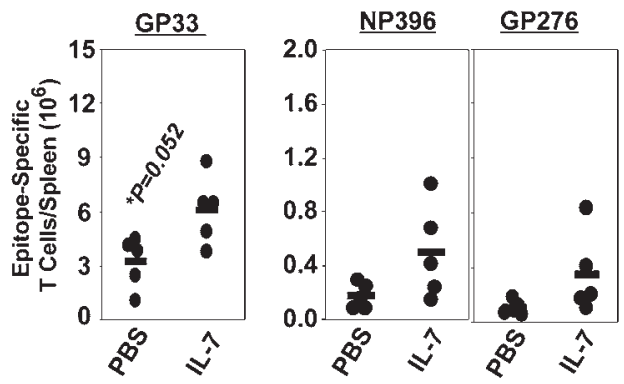

B
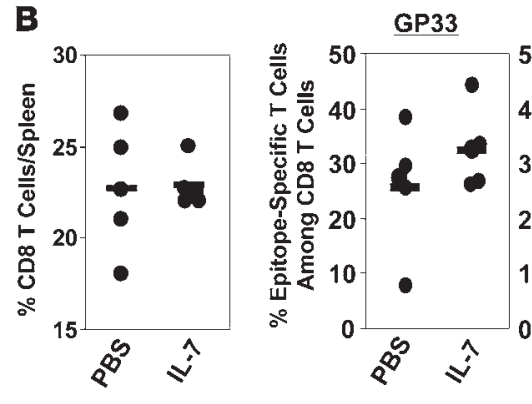

NP396

GP276

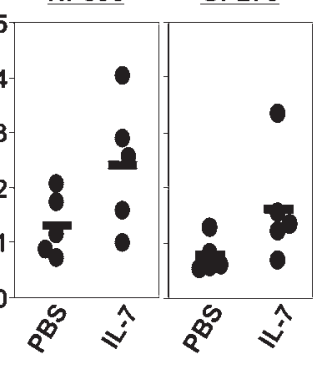

IL-7R is expressed in all peripheral CD8 T cells, except effector cells that are slated for deletion (8). Therefore, in theory, inflation of LCMV-specific memory CD8 T cells induced by IL-7 therapy during the contraction phase might be due to a total increase in CD8 $\mathrm{T}$ cells and/or to preferential effects on virus-specific CD8 T cells. Analyses of CD8 T cells immediately after cessation of IL-7 treatment on day 15 after infection indicated that the increased number of LCMV-specific CD8 T cells in IL-7-treated mice on day 15 could be attributed to a global increase in CD8 T cells coupled with preferential expansion of virus-specific CD $8 \mathrm{~T}$ cells. In contrast, at 28 days after IL-7 withdrawal, an enhanced number of LCMV-specific memory CD8 T cells in the spleens of IL-7-treated mice could not be linked to an increase in the total number of CD8 T cells. Instead, the increase in LCMV-specific memory CD8 T cells was epitope-specific and showed a strong correlation with elevated frequencies of GP33and GP276-specific CD8 T cells among splenocytes and total CD8 T cells. Collectively, these data suggest that IL-7 treatment during the contraction phase induces a transient global (nonspecific) increase in the number of CD8 T cells at the outset, but, subsequent to treatment withdrawal, cellular effects of IL-7 appear to preferentially enhance memory CD8 T cells of certain specificities. Perhaps, the selective enhancement of memory to certain LCMV epitopes might be related to the differentiation status and/or the proliferative history of the cell before IL-7 therapy. As in LCMV infection, IL-7 treatment of VV-infected mice during the contraction phase enhanced the number of virus-specific memory CD8 T cells following a transient spike in the total number of CD8 T cells. It would be interesting to understand the homeostatic mechanisms underlying the differences in the fate of CD8 T cells exposed to supraphysiological levels of IL-7 during an immune response. Apart from quantitative alterations in antigen-specific CD8 T cells, IL-7 also enhanced their intrinsic protective abilities; virus-specific CD8 T cells from IL-7treated mice controlled a persistent LCMV infection more effectively than did cells from PBS-treated mice. Improved control of LCMV infection by IL-7-exposed CD8 T cells correlated with enhanced effector function directly ex vivo and with antigen-induced proliferation in vitro and in vivo. It remains to be determined whether

\section{Figure 11}

IL-7 treatment during the contraction phase of the secondary response enhances CD8 T cell memory. One hundred days after LCMV infection, mice were challenged with recombinant Listeria monocytogenes (LM-GP33) that expresses the GP33 epitope of LCMV. LM-GP33infected mice were treated daily with IL-7 or PBS between days 5 and 15 after challenge. On day 34 after challenge, the number of CD8 T cells in the spleen specific to the indicated LCMV epitope (NP396, GP33, and GP276) was enumerated by intracellular staining for IFN- $\gamma$ as described in Methods. Flow cytometry data were analyzed to determine the percentages of CD8 T cells and IFN- $\gamma$-producing CD8 T cells in individual mice. (A) The total number of epitope-specific IFN- $\gamma$-producing CD8 T cells in the spleens of PBS- and IL-7-treated mice. (B) Percentages of CD8 T cells/spleen or percentages of epitope-specific CD8 T cells among total CD8 T cells in the spleens of PBS- or IL-7-treated mice. Note that the symbols represent data from an individual mouse.

IL-7-induced phosphorylation of FOXO transcriptional factors and downregulation of $\mathrm{p}^{27^{\mathrm{Kip}} 1}$ activity (42-44) augmented recall responses of $\mathrm{LCMV}$-specific CD8 T cells. It is important to note that IL-7 therapy-induced improvements in CD8 T cell memory are not restricted to acute viral infections, because exogenous IL-7 enhanced CD8 $\mathrm{T}$ cell memory and recall responses following immunization with a nonreplicating plasmid DNA. Thus, IL-7 might be used as an immunotherapeutic agent to bolster DNA vaccine-induced CD8 T cell memory in humans and animals.

It is well established that, after infection, long-term maintenance of antigen-specific memory CD8 T cells under steady state conditions requires IL-7 and IL-15 (10). According to the current paradigm, IL-7 is important for cell survival, and IL-15 is necessary for the proliferative renewal of memory CD8 T cells (10). However, distinct cellular effects are induced by IL-7 in a dose-dependent fashion: low concentrations of IL-7 (as seen in immune homeostasis) promote cell survival, whereas high concentrations of IL-7 (seen in lymphopenic conditions) induce proliferation (45). Accordingly, we found that exogenous administration of supraphysiological doses of IL-7 to LCMV-immune mice increased proliferation and led to a substantial increase in the number of memory CD8 T cells. However, within the subsequent 90 days, the number of memory CD8 T cells in IL-7-treated mice returned to pretreatment levels. This decay in cell numbers indicated that treatment with IL-7 alone does not result in long-term inflation of memory CD8 T cells. Nevertheless, these data show that IL-7 therapy can be used to bolster CD8 T cell memory and thereby enhance immunity, at least in the short term. What is the mechanism underlying the decay of memory CD8 T cell numbers in LCMV-immune mice after cessation of IL-7 therapy? The homeostatic mechanisms responsible for the remarkable reversion of memory CD8 T cell numbers to pre-IL-7 treatment levels are largely unknown. It is believed that the limited availability of prosurvival cytokines, such as IL-7, might impose a constraint on the size of T cell memory. However, this notion has its flaws because strong recall responses do increase the size of CD8 T cell memory. Alternatively, it is possible that stimulation of IL-7R signaling in the absence of additional signals will only promote cell cycle entry without programming daughter cells for long-term survival. Future experiments will test the influence of inflammation and/or antigenic stimulation during IL-7 supplementation on long-term CD8 T cell memory.

It was previously reported that the dynamics of primary and secondary CD8 $\mathrm{T}$ cell responses differ in many ways, including the relative durations of the expansion and contraction phases and the time required for effector to memory differentiation $(46,47)$. 
Here, we showed that IL-7 treatment during the contraction phase of a secondary CD8 $\mathrm{T}$ cell response led to a substantive increase in the number of memory CD8 T cells. Thus, IL-7 therapy during the contraction phase of primary or secondary CD8 $\mathrm{T}$ cell response augments CD8 T cell memory. Because heterologous infections have been shown to induce attrition of memory CD8 T cells, it will be interesting to test whether IL-7 therapy during such infections can protect against the attrition of CD8 T cell memory.

CD4 T cells influence several aspects of CD8 T cell responses, including the development of long-term CD8 T cell memory and protective immunity $(25,29,48)$. Although the focus of this manuscript is on CD8 T cells, it has to be noted that IL-7 treatment would affect other cell types, such as CD4 T cells, B cells, and dendritic cells. Therefore, it is possible that IL-7 treatment could modulate CD8 $\mathrm{T}$ cell homeostasis by direct and/or indirect mechanisms. We have begun to address this issue by performing preliminary analyses of the effects of IL-7 therapy on LCMV-specific CD4 $\mathrm{T}$ cells. The effects of IL-7 on CD4 T cell homeostasis appear to be largely similar to those of CD8 T cells. Administration of IL-7 during contraction but not during the expansion phase significantly increased the number of LCMV-specific memory CD4 T cells (S.G. Nanjappa and M. Suresh, unpublished observations). Similar to the effect on CD8 T cell memory, IL-7 treatment during memory phase temporarily enhanced the number of LCMV-specific memory CD4 T cells (S.G. Nanjappa and M. Suresh, unpublished observations). Future studies will determine the role of CD4 T cells in regulating the effects of IL-7 on CD8 T cell homeostasis.

What are the implications of our findings? Here, we systematically explored the effectiveness of IL-7 therapy during different phases of the $\mathrm{T}$ cell response as an adjunct to improve CD8 $\mathrm{T}$ cell memory. We provide strong evidence that administration of IL-7 during the primary antigen-driven expansion phase has minor effects on the magnitude of expansion, contraction, or immune memory. Thus, the use of IL-7 as an adjuvant at the time of immunization is unlikely to be beneficial. Instead, we showed that IL-7 therapy during the contraction phase of the primary $\mathrm{T}$ cell response to viral infections or DNA vaccine bolsters CD8 T cell memory and recall responses to antigen rechallenge. These findings suggest that exogenous administration of IL-7 during the contraction phase of the CD8 $\mathrm{T}$ cell response could be used as an immunotherapeutic modality to bolster vaccine-induced CD8 $\mathrm{T}$ cell memory and protective immunity. IL-7 administration to immune mice led to a substantial but transient increase in the number of antigen-specific memory CD8 T cells, which indicated that IL-7 could be used to temporarily boost preexisting CD8 T cell memory during disease outbreaks. In summary, the findings presented in this manuscript have implications for the clinical use of IL-7 to enhance immune memory following primary or booster vaccinations.

\section{Methods}

Mice. C57BL/6 mice were purchased from the National Cancer Institute. The generation and use of P14 TCR transgenic mice on the C57BL/6/ Thy1.1 background was described elsewhere (8). All mice were maintained under specific-pathogen-free conditions at the University of Wisconsin, and all animals were used in accordance with the strict guidelines of the institutional animal care committee.

IL-7 treatment. Recombinant human IL-7 was kindly provided by the National Cancer Institute or directly obtained from Cytheris Inc. Recombinant IL-7 was diluted in sterile PBS, and mice received daily i.p. injections of IL-7 at a dose of $5 \mu \mathrm{g} /$ mouse (17).
Virus and bacteria. Mice were infected with $2 \times 10^{5}$ PFU of the Arm strain of LCMV by i.p. injection. In some experiments, mice were challenged with $1 \times 10^{6} \mathrm{PFU}$ of LCMV-clone 13 by i.v. injection. The recombinant VV that express the GP of LCMV (VV-GP) was provided by Lindsay Whitton (Scripps Research Institute) (30). Mice were infected with $1 \times 10^{6}$ PFU of VV-GP i.p. Infectious LCMV in the tissues was quantitated by a plaque assay using Vero cells (49). In some experiments, LCMV-immune mice were challenged with $2 \times 10^{5}$ CFU of LM-GP by i.v. injection (50).

DNA immunization. The use of DNA plasmid encoding pCMV-NP was described previously $(32,51,52)$. C57BL/ 6 mice were injected once with $200 \mu \mathrm{g}$ of endotoxin-free pCMV-NP i.m. in the anterior tibialis muscles. Between days 14 and 21 after DNA immunization, mice were treated daily with PBS or IL-7 as described above.

Adoptive transfer of P14 TCR transgenic CD8 T cells. CD8 T cells $\left(2 \times 10^{5}\right.$ P14/+/+; Thy.1.1 $1^{+\mathrm{ve}}$ or Ly5.1 ${ }^{\text {ve }}$ ) were adoptively transferred into C57BL/6/ Thy1.2/Ly5.2 mice by tail vein injection and infected with LCMV 5 hours later. In some experiments, total T cells were purified from LCMV-infected P14 cell-recipient mice using T cell enrichment columns (R\&D Systems). Purified T cells from PBS- or IL-7-treated mice containing $9.4 \times 10^{5}$ P14/ Thy1.1 CD8 T cells were adoptively transferred into congenic Thy1.2/ C57BL/6 mice and challenged with LCMV-clone 13 twenty-four hours after cell transfer.

Flow cytometry. MHC class I tetramers were prepared and used as described previously (28). Briefly, single cell suspensions of splenocytes were stained with APC-labeled MHC tetramers, PE-labeled anti-CD8, and FITC-labeled anti-CD44 antibodies. In some experiments, antiCD62L, anti-CD127, anti-Thy1.2, anti-Thy1.1, anti-Ly5.1, and anti-Ly5.2 antibodies were used as costains with anti-CD8 and MHC I tetramers. To stain for intracellular Bcl-2 and $\mathrm{Bcl}-\mathrm{x}_{\mathrm{L}}$, splenocytes were stained for cell surface molecules and subsequently permeabilized and stained for intracellular proteins using the Cytofix/Cytoperm kit (BD Biosciences - Pharmingen). All antibodies were purchased from BD Biosciences Pharmingen except anti-CD127 and anti-Ly5.2 antibodies, which were purchased from eBioscience.

Intracellular cytokine staining. Splenocytes were stimulated for 5 hours with LCMV CTL epitope peptides in vitro, and the number of cytokine-producing CD8 T cells was quantitated by flow cytometry (28).

Assessment of in vivo proliferation of LCMV-specific CD 8 T cells with BrdU. The in vivo proliferation of virus-specific CD8 T cells was assessed by administering $\mathrm{BrdU}(0.8 \mathrm{mg} / \mathrm{ml})$ in drinking water. Splenocytes were stained with anti-CD8, anti-CD44 antibodies, and MHC Class I tetramers. Following surface staining, cells were permeabilized and stained with anti-BrdU antibodies (B44; BD) as described previously (28).

Quantitation of apoptotic cells directly ex vivo by annexin V staining. Splenocytes were stained with anti-CD8, MHC I tetramers, and annexin V (BD Biosciences - Pharmingen) directly ex vivo $(23,24)$. The percentages of annexin $V^{\text {hi }}$ LCMV-specific CD8 T cells were quantitated by flow cytometry.

Cytotoxicity assay. The MHC I-restricted cytotoxic activity of GP33-specific CD8 $\mathrm{T}$ cells was assessed directly ex vivo using a standard ${ }^{51} \mathrm{Cr}$ release assay (53).

Assessment of P14 CD8 T cell proliferation in vitro. Splenocytes were labeled with CFSE (Molecular Probes) and stimulated in vitro with $0.01 \mu \mathrm{g} / \mathrm{ml}$ of GP33 peptide for 60 hours as described previously (54). After being cultured, the cells were stained with anti-Ly5.1 and anti-CD8, and CFSE fluorescence was quantitated by flow cytometry.

Statistics. Experimental data were analyzed using commercially available statistical software (SYSTAT version 10.2; Systat Software, Inc.). Groups were compared by 2 -tailed Student's $t$ test, and significance was defined at $P<0.05$. 


\section{Acknowledgments}

We thank Katie Skell and Erin Hemmila-Plisch for excellent technical assistance, Anju Singh and Yumi Nakayama for help with the experiments, Chet Thomas for statistical analyses of data, and the National Cancer Institute for providing recombinant human IL-7. This work was supported by PHS grants AI48785, AI59804, and AI68841 to M. Suresh.

1. Seder, R.A., and Ahmed, R. 2003. Similarities and differences in CD4+ and CD8+ effector and memory $\mathrm{T}$ cell generation. Nat. Immunol. 4:835-842.

2. Kaech, S.M., Wherry, E.J., and Ahmed, R. 2002. Effector and memory T-cell differentiation: implications for vaccine development. Nat. Rev. Immunol. 2:251-262.

3. Ma, A., Koka, R., and Burkett, P. 2006. Diverse functions of IL-2, IL-15, and IL-7 in lymphoid homeostasis. Annu. Rev. Immunol. 24:657-679.

4. Fry, T.J., and Mackall, C.L. 2002. Interleukin-7: from bench to clinic. Blood. 99:3892-3904.

5. Schluns, K.S., Kieper, W.C., Jameson, S.C., and Lefrancois, L. 2000. Interleukin-7 mediates the homeostasis of naive and memory CD8 T cells in vivo. Nat. Immunol. 1:426-432.

6. Schluns, K.S., and Lefrancois, L. 2003. Cytokine control of memory T-cell development and survival. Nat. Rev. Immunol. 3:269-279.

7. Osborne, L.C., et al. 2007. Impaired CD8 T cell memory and CD4 $\mathrm{T}$ cell primary responses in IL7R\{alpha\} mutant mice. J. Exp. Med. 204:619-631.

8. Kaech, S.M., et al. 2003. Selective expression of the interleukin 7 receptor identifies effector CD8 T cells that give rise to long-lived memory cells. Nat. Immunol. 4:1191-1198.

9. Huster, K.M., et al. 2004. Selective expression of IL-7 receptor on memory T cells identifies early CD40L-dependent generation of distinct CD8+ memory T cell subsets. Proc. Natl. Acad. Sci. U. S. A. 101:5610-5615.

10. Surh, C.D., Boyman, O., Purton, J.F., and Sprent, J. 2006. Homeostasis of memory T cells. Immunol. Rev. 211:154-163.

11. Sasson, S.C., Zaunders, J.J., and Kelleher, A.D. 2006. The IL-7/IL-7 receptor axis: understanding its central role in T-cell homeostasis and the challenges facing its utilization as a novel therapy. Curr. Drug Targets. 7:1571-1582.

12. Snyder, K.M., Mackall, C.L., and Fry, T.J. 2006. IL-7 in allogeneic transplant: clinical promise and potential pitfalls. Leuk. Lymphoma. 47:1222-1228.

13. Rosenberg, S.A., et al. 2006. IL-7 administration to humans leads to expansion of CD8+ and CD4+ cells but a relative decrease of CD4+ T-regulatory cells. J. Immunother. 29:313-319.

14. Ehlken, H., Schadendorf, D., and Eichmuller, S. 2004. Humoral immune response against melanoma antigens induced by vaccination with cytokine gene-modified autologous tumor cells. Int. J. Cancer. 108:307-313.

15. Beq, S., et al. 2006. IL-7 induces immunological improvement in SIV-infected rhesus macaques under antiviral therapy. J. Immunol. 176:914-922.

16. Fry, T.J., et al. 2003. IL-7 therapy dramatically alters peripheral T-cell homeostasis in normal and SIV-infected nonhuman primates. Blood. 101:2294-2299.

17. Melchionda, F., et al. 2005. Adjuvant IL-7 or IL-15 overcomes immunodominance and improves survival of the CD8+ memory cell pool. J. Clin. Invest. 115:1177-1187.

18. Kieper, W.C., et al. 2002. Overexpression of interleukin (IL)-7 leads to IL-15-independent generation of memory phenotype CD8+ T cells. J. Exp. Med. 195:1533-1539.

19. Fry, T.J., Christensen, B.L., Komschlies, K.L., Gress, 174:6967-6973.
Received for publication March 5, 2007, and accepted in revised form November 28, 2007.

Address correspondence to: M. Suresh, 2015 Linden Drive, Department of Pathobiological Sciences, University of Wisconsin-Madison, Madison, Wisconsin 53706, USA. Phone: (608) 265-9791; Fax: (608) 263-0438; E-mail: sureshm@svm.vetmed.wisc.edu.
R.E., and Mackall, C.L. 2001. Interleukin-7 restores immunity in athymic T-cell-depleted hosts. Blood. 97:1525-1533.

20. Liu, N., et al. 2004. Serine protease inhibitor $2 \mathrm{~A}$ is a protective factor for memory $\mathrm{T}$ cell development. Nat. Immunol. 5:919-926.

21. Maraskovsky, E., et al. 1997. Bcl-2 can rescue T lymphocyte development in interleukin-7 receptor-deficient mice but not in mutant rag-1-/- mice. Cell. 89:1011-1019.

22. Zhang, N., and He, Y.W. 2005. The antiapoptotic protein $\mathrm{Bcl}-\mathrm{xL}$ is dispensable for the development of effector and memory T lymphocytes. J. Immunol.

23. Suresh, M., Singh, A., and Fischer, C. 2005. Role of tumor necrosis factor receptors in regulating CD8 T-cell responses during acute lymphocytic choriomeningitis virus infection. J. Virol. 79:202-213.

24. Wang, X.Z., et al. 2003. Virus-specific CD8 T cells in peripheral tissues are more resistant to apoptosis than those in lymphoid organs. Immunity. 18:631-642.

25. Williams, M.A., Holmes, B.J., Sun, J.C., and Bevan, M.J. 2006. Developing and maintaining protective CD8+ memory T cells. Immunol. Rev. 211:146-153.

26. Moniuszko, M., et al. 2004. Recombinant interleukin-7 induces proliferation of naive macaque CD4+ and CD8+ T cells in vivo. J. Virol. 78:9740-9749.

27. Mazo, I.B., et al. 2005. Bone marrow is a major reservoir and site of recruitment for central memory CD8+ T cells. Immunity. 22:259-270.

28. Murali-Krishna, K., et al. 1998. Counting antigenspecific CD8 T cells: a reevaluation of bystander activation during viral infection. Immunity. 8:177-187.

29. Fuller, M.J., et al. 2005. Cutting edge: emergence of CD127high functionally competent memory $\mathrm{T}$ cells is compromised by high viral loads and inadequate T cell help. J. Immunol. 174:5926-5930.

30. Whitton, J.L., Southern, P.J., and Oldstone, M.B. 1988. Analyses of the cytotoxic T lymphocyte responses to glycoprotein and nucleoprotein components of lymphocytic choriomeningitis virus. Virology. 162:321-327.

31. Tscharke, D.C., et al. 2005. Identification of poxvirus $\mathrm{CD} 8+\mathrm{T}$ cell determinants to enable rational design and characterization of smallpox vaccines. J. Exp. Med. 201:95-104.

32. Hassett, D.E., Slifka, M.K., Zhang, J., and Whitton, J.L. 2000. Direct ex vivo kinetic and phenotypic analyses of CD8(+) T-cell responses induced by DNA immunization. J. Virol. 74:8286-8291.

33. Rodriguez-Carreno, M.P., et al. 2005. Evaluating the immunogenicity and protective efficacy of a DNA vaccine encoding Lassa virus nucleoprotein. Virology. 335:87-98.

34. Yokoyama, M., Zhang, J., and Whitton, J.L. 1995. DNA immunization confers protection against lethal lymphocytic choriomeningitis virus infection. J. Virol. 69:2684-2688.

35. Hassett, D.E., Zhang, J., and Whitton, J.L. 1999. Plasmid DNA vaccines are effective in the absence of IFNgamma. Virology. 263:175-183.

36. Henson, S.M., Snelgrove, R., Hussell, T., Wells, D.J., and Aspinall, R. 2005. An IL-7 fusion protein that shows increased thymopoietic ability. J. Immunol. 175:4112-4118.
37. Franchimont, D., et al. 2002. Positive effects of glucocorticoids on $\mathrm{T}$ cell function by up-regulation of IL-7 receptor alpha. J. Immunol. 168:2212-2218.

38. Hand, T.W., Morre, M., and Kaech, S.M. 2007. Expression of IL-7 receptor alpha is necessary but not sufficient for the formation of memory CD8 T cells during viral infection. Proc. Natl. Acad. Sci. U. S. A. 104:11730-11735.

39. Pellegrini, M., et al. 2004. Loss of Bim increases T cell production and function in interleukin 7 receptor-deficient mice. J. Exp. Med. 200:1189-1195.

40. Opferman, J.T., et al. 2003. Development and maintenance of $\mathrm{B}$ and $\mathrm{T}$ lymphocytes requires antiapoptotic MCL-1. Nature. 426:671-676.

41. Hildeman, D.A., et al. 2002. Activated T cell death in vivo mediated by proapoptotic bcl-2 family member bim. Immunity. 16:759-767.

42. Barata, J.T., et al. 2004. Activation of PI3K is indispensable for interleukin 7-mediated viability, proliferation, glucose use, and growth of $\mathrm{T}$ cell acute lymphoblastic leukemia cells. J. Exp. Med. 200:659-669

43. Li, W.Q., et al. 2006. IL-7 promotes T cell proliferation through destabilization of $\mathrm{p} 27 \mathrm{Kip} 1$. J. Exp. Med. 203:573-582.

44. Jiang, Q., et al. 2005. Cell biology of IL-7, a key lymphotrophin. Cytokine Growth Factor Rev. 16:513-533.

45. Bradley, L.M., Haynes, L., and Swain, S.L. 2005. IL-7: maintaining T-cell memory and achieving homeostasis. Trends Immunol. 26:172-176.

46. Badovinac, V.P., Messingham, K.A., Hamilton, S.E., and Harty, J.T. 2003. Regulation of CD8+ T cells undergoing primary and secondary responses to infection in the same host. J. Immunol. 170:4933-4942.

47. Grayson, J.M., Harrington, L.E., Lanier, J.G., Wherry, E.J., and Ahmed, R. 2002. Differential sensitivity of naive and memory CD8+ T cells to apoptosis in vivo. J. Immunol. 169:3760-3770.

48. Williams, M.A., and Bevan, M.J. 2007. Effector and memory CTL differentiation. Annu. Rev. Immunol. 25:171-192.

49. Ahmed, R., Salmi, A., Butler, L.D., Chiller, J.M., and Oldstone, M.B. 1984. Selection of genetic variants of lymphocytic choriomeningitis virus in spleens of persistently infected mice. Role in suppression of cytotoxic T lymphocyte response and viral persistence. J. Exp. Med. 160:521-540.

50. Sun, J.C., Williams, M.A., and Bevan, M.J. 2004. $\mathrm{CD} 4+\mathrm{T}$ cells are required for the maintenance, not programming, of memory CD8 $+\mathrm{T}$ cells after acute infection. Nat. Immunol. 5:927-933.

51. Hassett, D.E., Zhang, J., Slifka, M., and Whitton, J.L. 2000. Immune responses following neonatal DNA vaccination are long-lived, abundant, and qualitatively similar to those induced by conventional immunization. J. Virol. 74:2620-2627.

52. Martins, L.P., Lau, L.L., Asano, M.S., and Ahmed, R. 1995. DNA vaccination against persistent viral infection. J. Virol. 69:2574-2582.

53. Suresh, M., et al. 2003. Complement component 3 is required for optimal expansion of CD8 T cells during a systemic viral infection. J. Immunol. 170:788-794.

54. Kaech, S.M., Hemby, S., Kersh, E., and Ahmed, R. 2002. Molecular and functional profiling of memory CD8 T cell differentiation. Cell. 111:837-851. 